\title{
Thermodynamic Characterization of Planar Shapes and Curves, and the Query of Temperature in Black Holes
}

\author{
Güngör Gündüz \\ Orta Doğu Teknik Üniversitesi, Ankara, Turkey \\ Email:ggunduz@metu.edu.tr
}

How to cite this paper: Gündüz, G. (2021) Thermodynamic Characterization of Planar Shapes and Curves, and the Query of Temperature in Black Holes. Journal of Applied Mathematics and Physics, 9, 2004-2037.

https://doi.org/10.4236/jamp.2021.98131

Received: July 22, 2021

Accepted: August 28, 2021

Published: August 31, 2021

Copyright $\odot 2021$ by author(s) and Scientific Research Publishing Inc. This work is licensed under the Creative Commons Attribution International License (CC BY 4.0).

http://creativecommons.org/licenses/by/4.0/ (c) (i) Open Access

\begin{abstract}
The purpose of this research is to characterize shapes in thermodynamic terms, namely, in terms of total energy, dissipative energy, entropy, and temperature. As case studies, polygons and some well-known curves were taken, and they were characterized using physical terms. The relation between entropy and curvature was elucidated, and the black hole surface gravity and temperature were criticized and reinterpreted from this point of view. Particular energy attributions were evaluated by comparing the position of any edge of a polygon (i.e. its angle with the horizontal axis) with a broken crystal surface. Energies of all edges were added up at all positions between $0^{\circ}-360^{\circ}$. In regular polygons, the total energy decreases with the increase of the number of edges. Entropy increases in the reverse order, and the increase of the number of edges increases entropy. It implies that the circle has the lowest energy but the highest surface entropy. In curves (circle, sine-curve, spiral, and exponential curve), the total energy, dissipative energy, and entropy all depend on amplitude and also on specific variables. Black hole entropy expressed in terms of the surface area is a configurational entropy and not thermal entropy; therefore, it does not involve a varying temperature term. The surface gravity of a black hole is connected to acceleration and thus to curvature. To relate it with the temperature needs to be reinterpreted, because, surface gravity behaves like an attractive force not exactly like temperature. Hawking radiation is still possible, but the black hole does not get warmer as it evaporates. Material loss from the black hole gets faster as its radius decreases due to the curvature effect, i.e. by a mechanism similar to the Gibbs-Thomson effect.
\end{abstract}

\section{Keywords}

Polygons, Curves, Viscoelasticity, Energy, Entropy, Temperature, Black Hole 


\section{Introduction}

Establishing a relation between entropy and geometry has been a tough issue in science, because, geometry concerns with shape, size, relative positions of figures, and also the type of space whether it is flat or curved, whereas thermodynamics deals with work, heat, entropy, and temperature which are dynamical properties. Although their domains look quite different from each other they have a striking similarity, essentially in the descriptions of the surface properties of polygons and the phase rule in thermodynamics. Any convex polyhedron's surface has Euler's topological characteristics $V-E+F=2$, where $V, E$, and $F$ are respectively the number of vertices, edges, and faces. In thermodynamics, the Gibbs phase rule is given by $F-C+P=2$, where $F, C$, and $P$ refer to the degrees of freedom, the number of the constituent components, and the number of phases, respectively. Gibbs developed the graphical properties of equilibrium phases, and his internal energy function may be represented as a surface in a multidimensional Cartesian coordinate system. Weinhold argued that thermodynamics has conjugate relations resembling the equations of classical mechanics, such that temperature $(T)$ can be described as being conjugate to entropy $(S)$, and pressure with a negative sign $(-P)$ to volume $(V)$. The internal energy $(U)$ is a function of entropy, volume, and chemical components $\left(N_{1}, N_{2}, \ldots\right)$, and it can be represented as a surface in a multidimensional Cartesian coordinate system [1]. He claimed that Riemannian geometry or some abstract geometry may provide additional insights into thermodynamic behavior. This idea had been taken up by others to represent thermodynamics by Riemannian manifolds. In these efforts, the interactions were expressed in terms of curvature tensor, such that the curvature of internal energy can be expressed in terms of the second derivatives of extensive variables [1] [2] [3] [4]. The second derivative refers to the curvature of the surface of equilibrium states, and dissipative reactions or changes give rise to curvature [5]. Its convexity warrants the entropy principle. There have been numerous types of definitions of entropy in literature, and entropy usually has an intimate relation with intrinsic and extrinsic geometry of surfaces or shapes [6].

The shape of an object has no place in physical laws concerning the dynamical behavior of objects such as in Newton's force equation, nor in momentum and energy equations where we are concerned with mass only not with its shape. However, every object in the physical world has a shape, because, in Aristotle's words every physical entity has a boundary that imparts a shape to it; only metaphysical and non-physical entities have no boundaries. Anything which has a shape can be characterized by its energy content and its shape entropy. The shape of objects has become an important field of study concurrently with chaos theories, and especially after Maldelbrot's revolutionary fractal concept [7].

Entropy drives the phase behaviors of spheres, rods, suspensions, emulsions, etc., and yields ordered shapes at larger scales. The shape entropy becomes important when the intrinsic interactions start to dominate at moderate densities 
[8]. The entropy of shapes revealed as the interface between two phases can be determined by using discrete formulation but it can be extended to continuous formulation [9]. The fragmentation entropy of spiral tilings can also be calculated by the same method [10]. The Shannon entropy denotes the information content in a system. Based on this formulation the curvature of an object's boundary may also be used as an information measure [11]. In other words, curvature also may give information about the shape of an object [12]. By the same token, we may ask if known curvature can define temperature also for our system? This question is very difficult to answer, because, the only definition of temperature is given by Boltzmann for ideal gases in terms of translational average kinetic energy i.e., $(1 / 2) m v^{2}=(3 / 2) m v^{2} k T$. In thermodynamics, the temperature was expressed as the inverse of the rate of change of entropy with internal energy. If we can determine the entropy of geometric objects, can we also define temperature in terms of geometric variables? These questions will be tackled in this research work.

Another point to mention is that the entropy concept is also used as a measure of aesthetics [13]. There is an intimate relation between art (or aesthetics) and entropy [14] [15]. The second law of thermodynamics finds application to quantify graphic complexity and aesthetic optimality [16]. Polygons, besides their characterization by edges and angles, can be characterized also by their aesthetic measures. The first concrete procedure to establish a measure for the aesthetics of geometric shapes was done by Birkhoff [17]. He defined the ratio of order (O) to complexity $(\mathrm{C})$ as the aesthetic measure $(\mathrm{M})$, such that $\mathrm{M}=\mathrm{O} / \mathrm{C}$. He used terms related to the number of symmetry axis. Although square and rhombus have equivalent number of symmetry axis Birkhoff claimed that rhombus is less aesthetic than square, and he introduced a non-physical term into his equation called a non-pleasing parameter. Can entropy be used to explain the aesthetic difference between a square and rhombus without using Birkhoffs "non-pleasing parameter"? This point also will be shortly elaborated.

All the arguments made above were treated in this work using energy and entropy attributes of shapes. It is possible to characterize trigonometric shapes and curves quantitatively in thermodynamic terms like energy and entropy. This work aims to develop some procedures to find out total energy, dissipative energy, entropy, and temperature-like attributes of planar geometric shapes, like triangles and higher polygons, and also of some curves like a circle, sine-curve, spiral, and exponential curve. The theory of viscoelasticity will be utilized to attribute energies to trigonometric shapes and curves. The outcomes of these studies were then applied to characterize the entropy and temperature of Schwarzschild black holes.

\section{Surface Energy Attributes}

The difference between square and rhombus is that the internal angles of the rhombus are different than $90^{\circ}$. If we think of a rhombus with internal degrees 
of $90^{\circ}$ then it is nothing but a square rotated by $45^{\circ}$, and they are mathematically the same object, but from a physical point of view, they have different properties. Whether the surface is a rhombus or rotated square, each is different than a square from a physical point of view if they represent crystalline surfaces. To visualize this, consider two solid specimens both cut out from a bulky material one is in the shape of a cube and the other is in the shape of a pyramid. From the material physics point of view, we can ask if the surface of cube and pyramid have identical properties even though both are fine-polished. The answer would be simply negative, because, they are cut out at different angles. Polishing could be theoretically achieved at best to the unit cell size of the crystal. Three cases are shown in Figure 1 where each tiny grid square represents a unit cell size. Three different surfaces are produced depending on different cutting angles, these are, square produced by applying $0^{\circ}$ and/or $90^{\circ}$ of cutting angle rotated square produced by $45^{\circ}$ of cutting angle, and finally rhombus produced by an arbitrary cutting angle.

In Figure 1(a) all cells are aligned smoothly with respect to background geometry or say to a referenced crystal surface, but in Figure 1(b) the surface is inclined by $45^{\circ}$. That is, it represents a cross-cut view where the smooth crystal surface was cut at $45^{\circ}$ such that a new surface was created at each unit cell compared to Figure 1(a). In Figure 1(c) the cutting angle is something other than $45^{\circ}$. In material science, this figure depicts misorientation between the background (or reference) lattice and the lattice of an object. Depending on the angle between the two crystal sites at the interface one may classify them as the low- or high-angle boundaries. However, we will not do crystal orientation analysis here but concentrate only on the effect of angle of an edge such as that of Figure 1(b) \& Figure 1(c) with respect to the background square lattice in this work.

According to the Gibbs law, any surface no matter whether it is a droplet or crystal arranges itself in a manner to minimize its surface Gibbs free energy. In other words, a shape with low surface energy is achieved at the equilibrium state. According to Wulff, the length of a vector drawn from the center normal to the crystal face will be proportional to the surface energy of that face. It was proved years later that this proposal was scientifically true. The determination of the

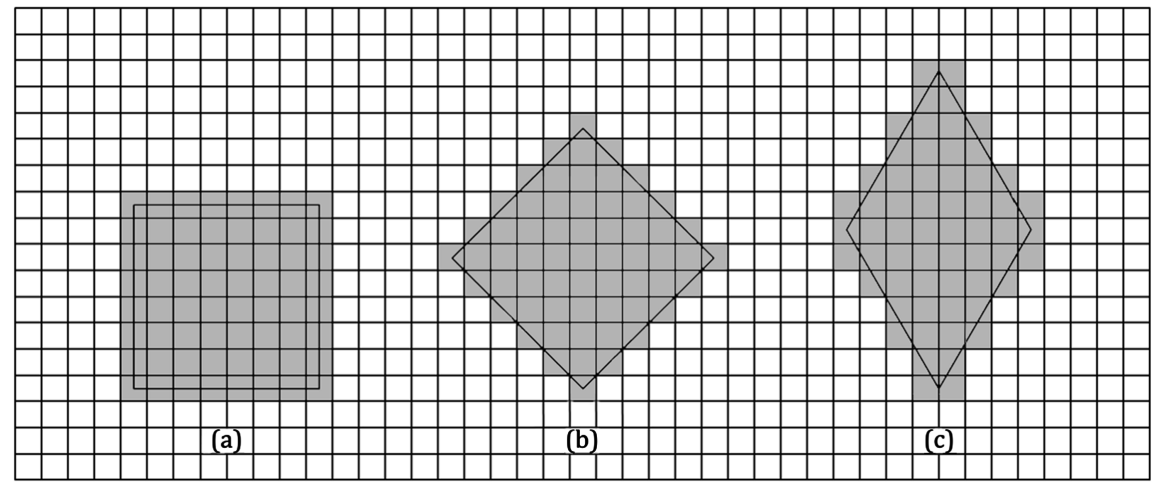

Figure 1. Surface of, (a) square, (b) rotated square, (c) rhombus. 
equilibrium shape of an object is called Wulff construction, and it finds a vast number of applications in applied sciences, and in theoretical studies as well [18]-[26]. The knowledge of surface energies with Miller indices provides knowledge about the shape of the crystal. However, in this research work, we will not use the Wulff construction to figure out the shapes of polygons, but we will rather use the shape energies of some simple polygons and also of curves, and then elucidate the associated shape entropies using the laws of thermodynamics.

In Figure 2, a general cross-sectional view of the broken surface of a crystal is shown, and only one edge is considered there, the second dimension was omitted. Each tick denotes say the edge of the unit cell of a broken surface of the crystal. Ticks are supposed to exist on the entire broken surface. Figure 2 may help to specify interfacial energy, meanwhile, it also may form the basis for the energy of the edges of geometric shapes. The amount of energy needed to create a surface is proportional to the surface area. The energy attributes can be evaluated by considering the surface energy along with both horizontal and vertical directions [27]. The calculations are limited to the ranges indicated by the double-headed arrows in Figure 2 where the ticks denote the boundaries.

The number of broken bonds along the horizontal (i.e. $x^{-}$) and vertical (i.e. $y^{-}$) directions can be found out from the geometry of the shape. The edge of a unit cell is given by " $d$ ", therefore the number of unit cells is inversely proportional to $d$ and directly proportional to the length $\ell$.

$$
\begin{aligned}
& \text { \# of broken bonds along } x \text {-direction }=\frac{\ell}{d} \cos (\theta) \\
& \text { \# of broken bonds along } y \text {-direction }=\frac{\ell}{d} \sin (\theta)
\end{aligned}
$$

Their sum gives the total number of bonds along both directions,

$$
\text { total \# of broken bonds }=n_{d}=\frac{\ell}{d}(\cos (\theta)+\sin (\theta))
$$

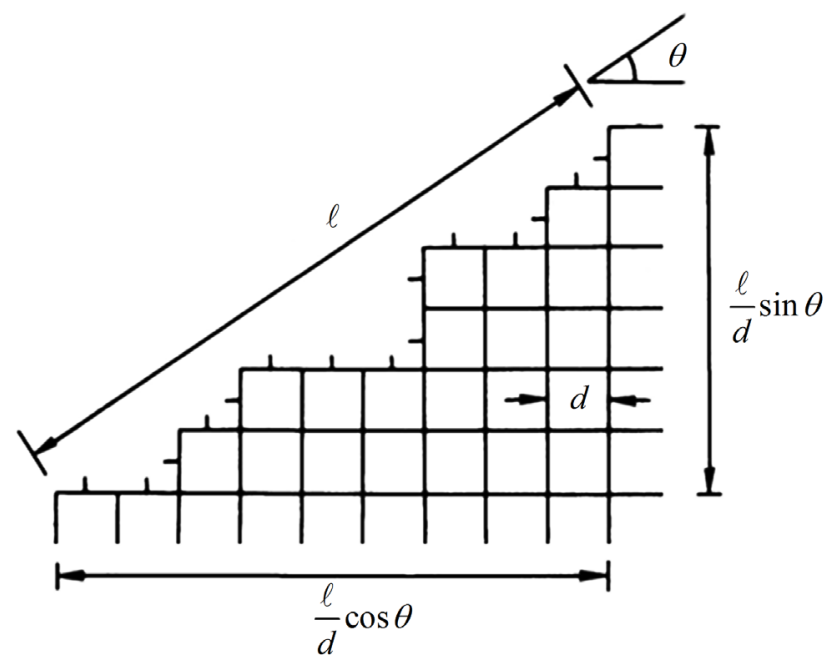

Figure 2. Surface pattern. 
Now let us assign a unit energy of $\varepsilon / 2$ to each bond. Then the total energy of created surfaces can be given by,

$$
E=\frac{\varepsilon}{2} \frac{\ell}{d}(\cos (\theta)+\sin (\theta))=\frac{\varepsilon \ell}{\sqrt{2} d} \cos (\theta-\pi / 4)
$$

There are two limiting energies, one is the minimum and the other maximum. The former occurs when $\theta=0^{\circ}$, and the other at $\theta=45^{\circ}$, such that,

$$
\begin{aligned}
& E_{\min }=\frac{\varepsilon}{2} \frac{\ell}{d} \\
& E_{\max }=\frac{\varepsilon \ell}{\sqrt{2} d}
\end{aligned}
$$

The values of $\varepsilon$ and $d$ are somehow arbitrary, so we can rearrange Equation (5) as,

$$
E\left(\frac{d}{\varepsilon}\right)=\frac{\ell}{2}(\cos (\theta)+\sin (\theta))=\frac{\ell}{\sqrt{2}} \cos (\theta-\pi / 4)
$$

The $45^{\circ}$ is a critical angle and we get the maximum number of broken bonds along the vertical axis at this angle. Below or above $45^{\circ}$ the broken bonds decrease in number. It can be better seen pictorially in Figure 3, where all lengths are of equal length.

We have $\theta=45^{\circ}$ for (d) in Figure 3, and it has the maximum energy given by Equation (7). The flat horizontal and vertical lengths, which are (a) and (f) respectively, have the minimum energy given by Equation (6). Similarly, (b) and (e) also have equal energies. The energy of (c) is between that of (b) and (d). Either (a) or (f) represents a smooth surface while others represent surfaces at different distortions.

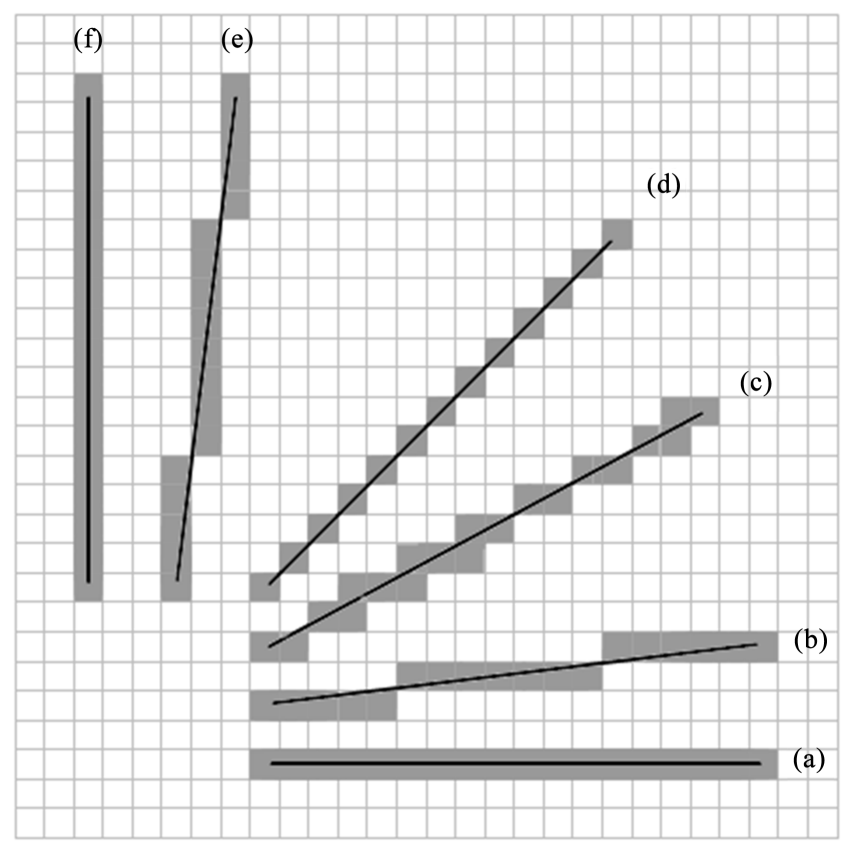

Figure 3. Lengths at different inclinations. 


\section{Surface Energy Attributes of Geometric Shapes}

\subsection{Triangles}

Let us form a triangle by pushing (a) up in Figure 3 until it touches (b) on the left to form a corner. Then the right ends of (a) and (b) can be joined by adding an edge. If we had $1^{\circ}$ tilted (a) and also $1^{\circ}$ titled (b) at the beginning, we would form the same triangle tilted by $1^{\circ}$. This second triangle is nothing but $1^{\circ}$ rotated shape of the former one. However, the second triangle has different surface properties than the first one as the tilting changes the surface properties of the edges. The surface energy can be found out by calculating energy using Equation (8) for each edge and summing them up. The rotation is performed in a counterclockwise direction by $1^{\circ}$ increase in each step until we stop at $360^{\circ}$. The energies of edges are summed up to find the total energy at each step.

Triangles with different shapes exhibit different energies as they are rotated. The types of triangles studied are shown in Figure A1 in Appendix A. Their areas are all equal, and it was arbitrarily taken to be 3600 units. The lengths of edges are shown on the edges.

In Figure 4 the change of the energy of the acute scalene triangle of which all angles and all edge lengths are different from each other is shown. In Figure A1 the angle of base $\mathrm{AB}$ is $\theta_{\mathrm{BA}}=0^{\circ}$, whereas those of $\mathrm{BC}$ and $\mathrm{AC}$ are $\theta_{\mathrm{BC}}=-60.95^{\circ}$, and $\theta_{\mathrm{CA}}=71.57^{\circ}$, respectively. These angles are found from the slopes of the edges. The very initial energy is calculated under these conditions, and in the next step the angle of $\mathrm{AB}$ is increased by $1^{\circ}$, and the others also change accordingly, thus we get $\theta_{\mathrm{BA}}=1^{\circ}, \theta_{\mathrm{BC}}=-59.95^{\circ}$, and $\theta_{\mathrm{CA}}=72.57^{\circ}$, respectively.

In the calculations, $d / \varepsilon$ was arbitrarily taken to be unity in Equation (8). The notations $\mathrm{AB}, \mathrm{BC}$, and $\mathrm{CB}$ in the legend of Figure 4 refer to the edges. $\mathrm{AB}$ is the shortest as seen from Figure $A 1$, so it generally has the lowest energy values as seen from the very low curve in Figure 4. Similarly, BC is the longest and it generally exhibits the highest values.

The change of total energy for all triangles is shown in Figure 5. The top curve in Figure 4 shows that the pattern repeats itself for every $90^{\circ}$ of rotation. For better visual inspection the first two quadrants were displayed in Figure 5.

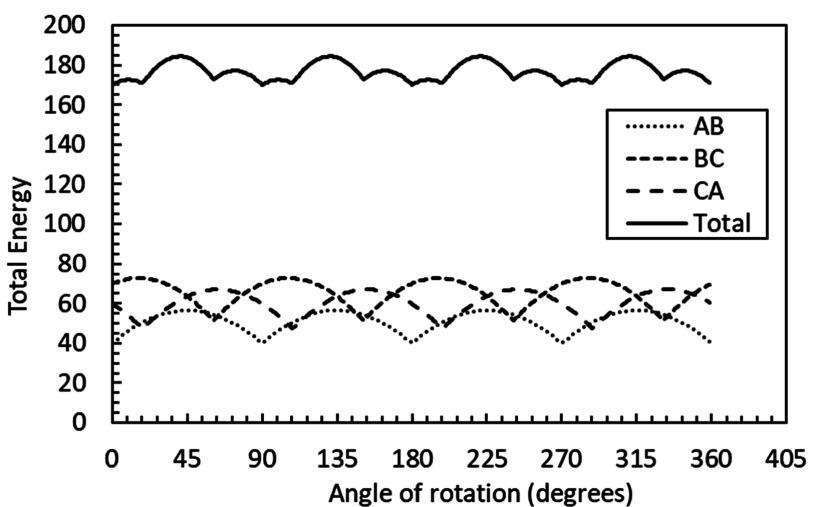

Figure 4. Change of energy of acute triangle with $\theta$, where $\mathrm{AB}, \mathrm{BC}$, and $\mathrm{CA}$ denote the edges. 


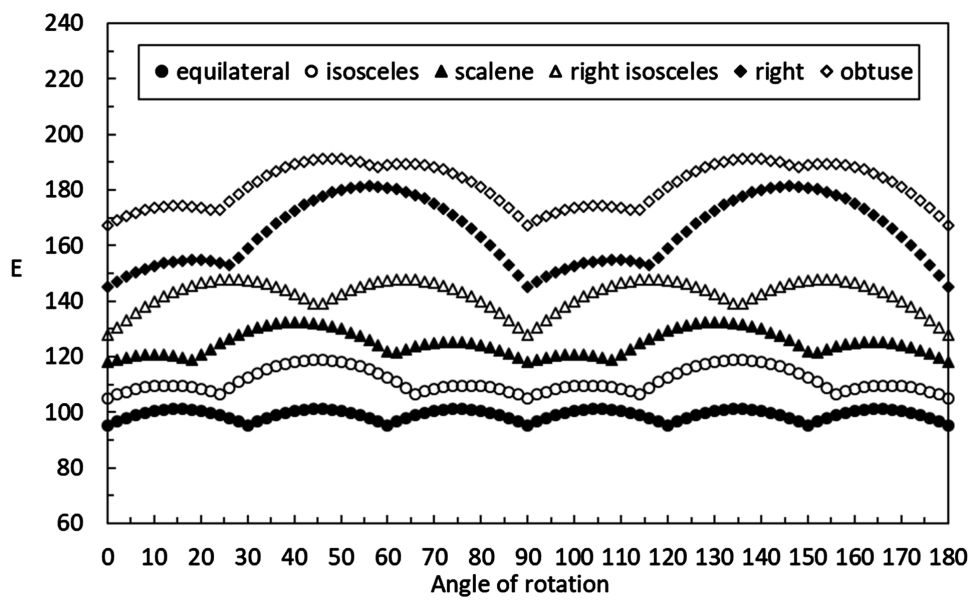

Figure 5. Change of energy of triangles with $\theta$.

As it is seen from Figure 5 equilateral triangle has the lowest total energy while obtuse has the largest energy in all degrees of rotation. Equilateral has the simplest pattern having the highest number of symmetry axes. It repeats itself for every $30^{\circ}$, i.e. the pattern repeats itself three times in each quadrant. Between $0^{\circ}-90^{\circ}$ right triangle and right isosceles triangle have two peaks, and each peak is symmetric in the right isosceles. Other triangles have three peaks in this quadrant.

The equilateral has the lowest energy while the obtuse has the highest. In all cases, the energy exhibits a minimum at $0^{\circ}, 90^{\circ}$, and $180^{\circ}$, because, these are the cases given by either (a) or (f) in Figure 3 for one of the edges. For equilateral and isosceles the energy becomes maximum at $45^{\circ}$ and $135^{\circ}$ (i.e. at odd-number multiples of $45^{\circ}$ between $0^{\circ}-180^{\circ}$ ). The maximum occurs usually close by $45^{\circ}$ in other cases except at right isosceles.

\subsection{Higher Polygons}

The energies of the rectangle and the regular polygons from square to octagon are given in Figure 6. The energy in each case was found out by calculating the energy of each edge of the polygon and summing up for all edges. The total energy was found from the sum of all energy values between $1^{\circ}-360^{\circ}$. Their total energies change as; rectangle: 55,919.26, square: 55,002.55, pentagon: 52,416.73, hexagon: 51,185.65, heptagon: 50,494.61, and octagon: 50,062.22 units. Rectangle has the highest energy. This might be because a rectangle has two longer sides, which make a larger contribution to energy.

In regular polygons (i.e. equal-sided polygons, rectangle not included) the energy decreases as the number of edges increase from square to octagon. The energies of the pentagon, hexagon, and heptagon are close to each other while square has the largest and octagon the smallest energy as seen from Figure 5.

If we keep increasing the number of edges of polygons indefinitely we finally get a circle. We can say that circle has the lowest energy than all polygons of the same area as the energy decreases with the increasing number of edges. 


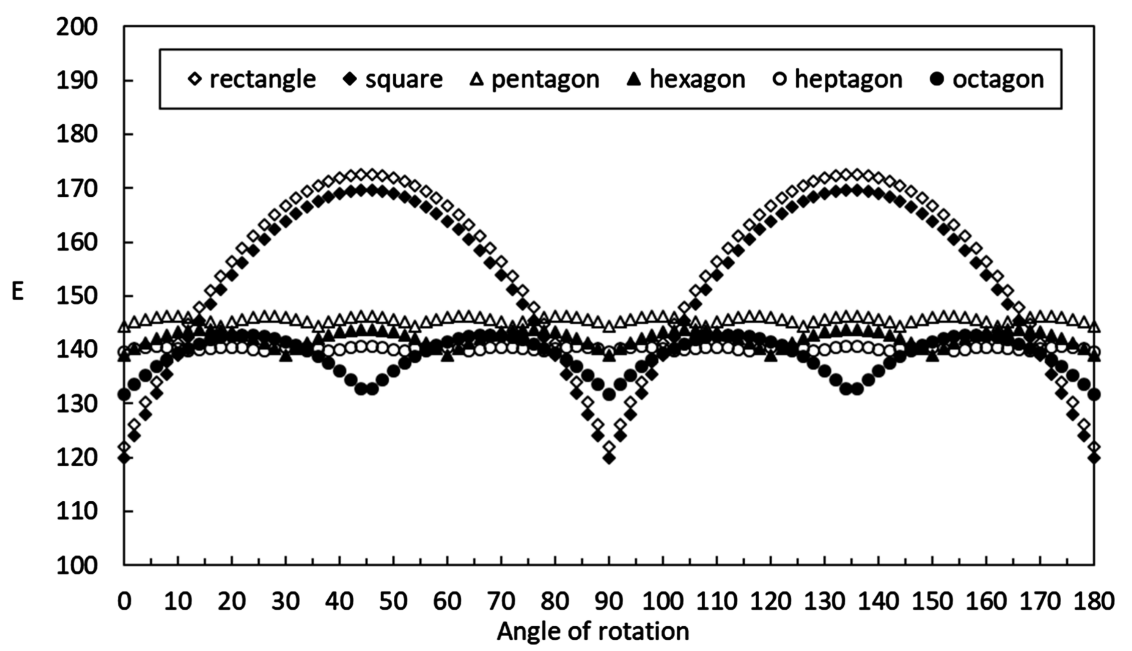

Figure 6. Change of energy of higher polygons with $\theta$.

\section{Conservative and Dissipative Energies}

In Figure 3 as we go from (a) to (d) through (b) and (c) there occurs new distortions in each case, and (a) is finally transformed into (d). In the vocabulary of material science, it is said that (a) is deformed into (b) and (b) is deformed into (c), and so on. The measure of deformation is the number of new bonds created along the vertical direction. In the theory of viscoelasticity, if an object is deformed under a force or stresses $(\tau)$, the stress is split into two components, one is along the direction of the applied stress and the other vertical to it. For instance, consider a semisoft partly deformable material that is pushed by applying a force from one end along its axial direction as seen in Figure 7(a).

The object responds in two ways; 1 ) it moves along the direction of force which is called "in-line" motion, and 2) it deforms in a direction vertical to the direction of applied force, and it is called "out-of-line" motion. The force (or stress) $(\tau)$ is split into its components along both directions. The $\tau_{\|}$denotes the parallel or in-line component of stress vector, and $\tau_{\perp}$ denotes the vertical or out-of-line component of stress as indicated in Figure $7(b)$. Note that the in-line motion is associated with conservative energy and the out-of-line motion with dissipative energy. In thermodynamics, the conservative energy is called work energy whereas dissipative energy is called heat energy. Here, we are not interested in the magnitude of stress as we are not dealing with a dynamic system. Whatever happens results in a change in the shape of the object and we are interested only in the surface properties. The energy and entropy attributes are related only to surface patterns.

Equation (8) gives the sum of these energies; the cosine component denotes the work-like energy and the sine component denotes the heat-like energy associated with the surface. Since the entropy of a system is connected to its dissipative energy we can investigate how the entropy or randomness in geometric shapes can be expressed quantitatively. It is explained in Section 5 . The heat-like or dissipative energy $(q)$ can be simply deduced from Equation (8), such that, 


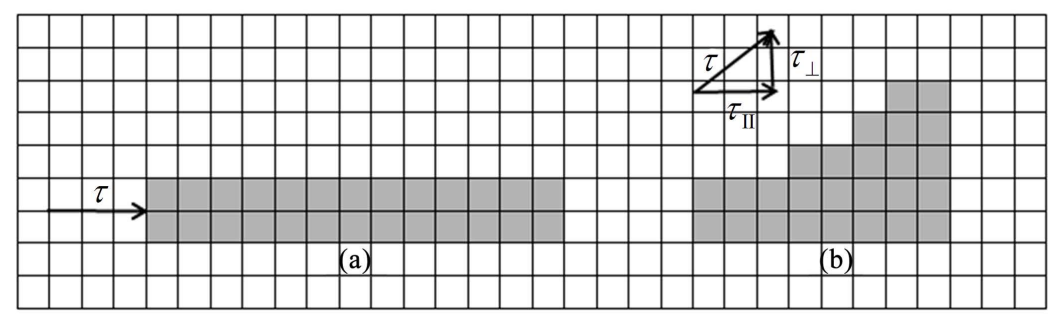

Figure 7. Deformation and stress components, (a) Initially, (b) After deformation.

$$
q\left(\frac{d}{\varepsilon}\right)=\frac{\ell}{2} \sin (\theta)
$$

The calculations can be simply done by using Equation (9) by changing $\theta$ by $1^{\circ}$, and the total $q$ can be found out from the summation of $q$ values of each edge of a polygon as done before in calculating the total energy $E$. Note that we must have $\theta \leq 45^{\circ}$, otherwise, the axes must be changed, i.e. the calculation must be done using $\sin (\theta) \rightarrow \sin (90-\theta)=\cos (\theta)$. Again, $d / \varepsilon$ was arbitrarily taken to be unity in Equation (9).

\subsection{Triangles}

The changes of dissipative energies $(q)$ of triangles with $\theta$ are shown in Figure 8.

The curves heavily overlapped with each other, therefore, only the changes between $0^{\circ}-180^{\circ}$ were presented. In all cases, $q$ reaches its maximum at $45^{\circ}$ and $135^{\circ}$, and in an equilateral triangle the peak occurs at every multiple of $45^{\circ} / 2$. The right triangle exhibited the largest magnitude of difference between its minimum and maximum values. The scalene and isosceles had similar patterns because their heights had been taken to be equal. The two tiny minima between $0^{\circ}-90^{\circ}$ occur at the same height in isosceles but at different heights in scalene. The change of $q$ with $\theta$ displayed a smooth curve in right isosceles, because, the angles between the hypotenuse and the other edges are $45^{\circ}$, and the contribution to $q$ used to change at appropriate proportions on rotation.

\subsection{Higher Polygons}

The dissipative energies of higher polygons (i.e. other than triangles) from rectangle to octagon are given in Figure 9. The amount of $q$ decreases in the order of, rectangle: 27,959.63, square: 27,501.28, pentagon: 26,208.37, hexagon: 25,592.82, heptagon: $25,247.31$, and octagon: $25,031.11$. This is the same order as in the case of the change of $E$. The rectangle has the highest dissipative energy. This might be due to the reason that rectangle has two longer sides than the square as mentioned before, and long edges make a larger contribution to $q$ when .

The visual inspection shows that the amplitude of each repeating pattern is minimum in the heptagon in Figure 6, and it increases as heptagon, pentagon, hexagon, octagon, and square. In Figure 9 , the order goes as heptagon, pentagon, octagon, hexagon, and square, where hexagon and octagon are switched 

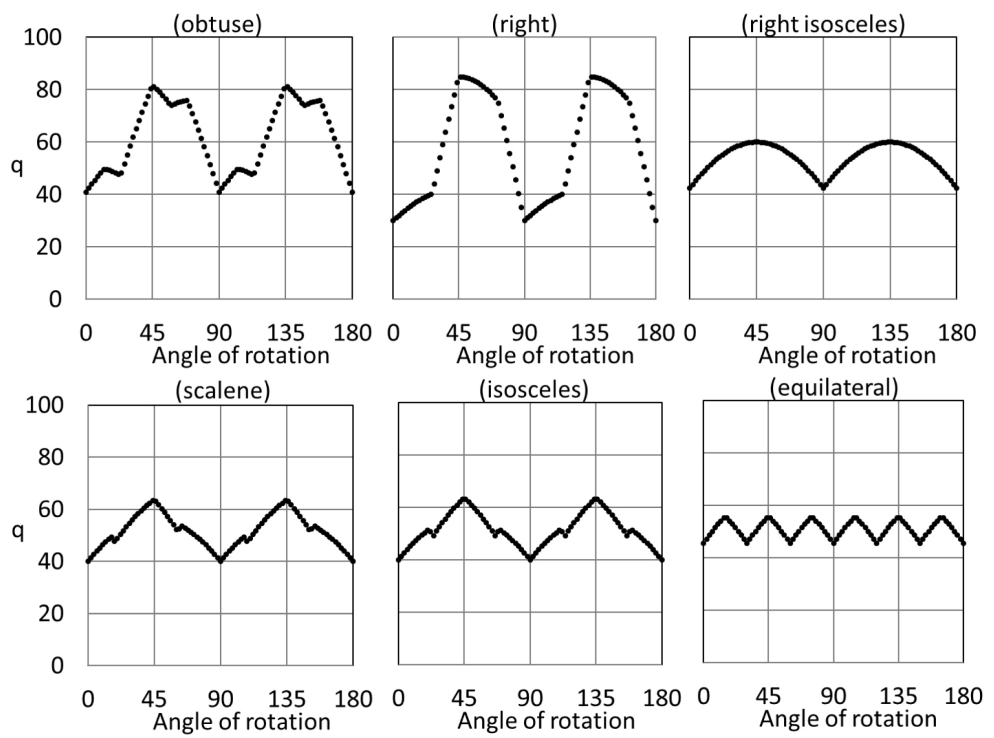

Figure 8. Change of dissipative energy of triangles with $\theta$.
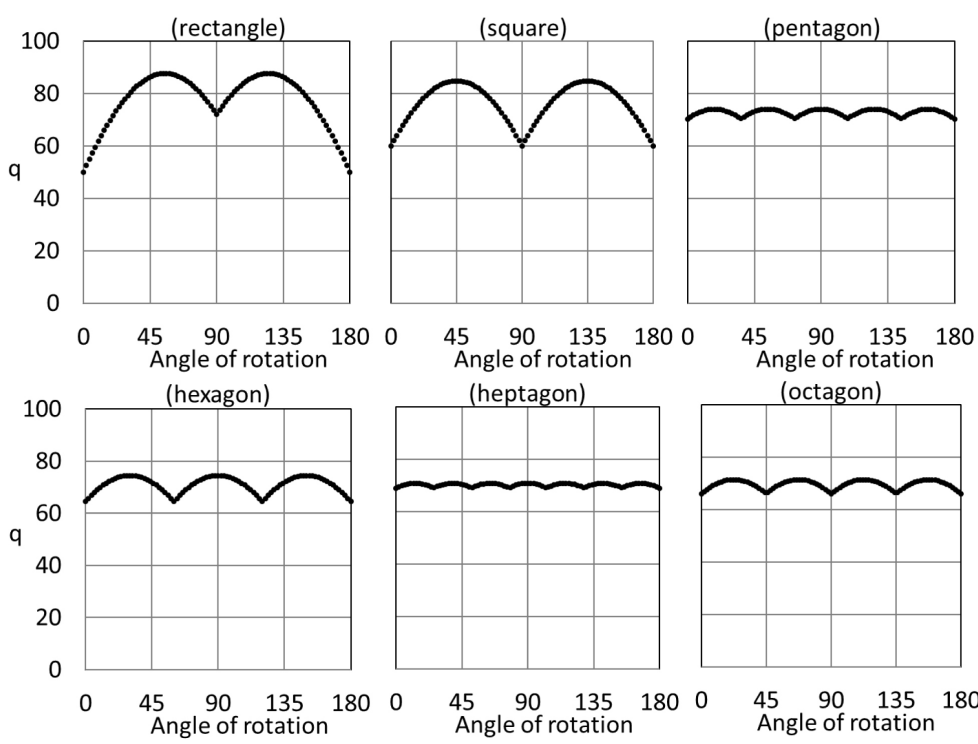

Figure 9. Change of dissipative energy of higher polygons with $\theta$.

compared to the order displayed in Figure 6. As the number of edges further increases the patterns likely get quite smoother.

\section{Entropy}

The definition of entropy as degradation of energy in classical thermodynamics leads to some unconformity, because, it has applications in some systems where there is no heat or energy exchange such as mixing of two miscible liquids at the same temperature. Boltzmann proved in his $\mathrm{H}$-theorem that entropy is a measure of the increase of randomness in a system. Boltzmann defined also the temperature in terms of kinetic energies of randomly colliding molecules, i.e. $(1 / 2) m v^{2}=(3 / 2) m v^{2} k T$, where $k$ is the Boltzmann constant, and it can be ex- 
pressed in terms of gas constant $R$ and the Avogadro number. In simple monoatomic ideal gas the heat capacity at constant volume processes is simply given by $(3 / 2) R$. In constant volume processes there is no work term, and the change in heat energy $(\mathrm{d} q)$ simply becomes equal to the change in internal energy, such that $\mathrm{d} q=(3 / 2) R \mathrm{~d} T$. For such systems, the change of entropy $(\mathrm{d} S)$ is simply expressed by $\mathrm{d} S=(3 / 2) R \mathrm{~d} T / T$. In other words, entropy change is given in terms of $d T / T$, that is in terms of the fractional change of random kinetic energy.

So far, we kept using thermodynamic terms and now we can make a paradigmatic change in our vocabulary while talking about the entropy of shapes. Entropy defined in terms of temperature as in classical thermodynamics is not appropriate for geometrical shapes. It is better to go with the Boltzmann-Shannon entropy where entropy can be determined from the prevailing states of a system. The associated temperature can then be evaluated after finding entropy. In this view, each square in Figure 2 corresponds to an "area state" and each broken bond corresponds to an "interface state". The elementary square size (i.e. $d^{2}$ ) multiplied by the number of squares (i.e. $N_{\mathrm{sq}}$ ) gives the total area. Similarly, the "NumberLines" at the boundary multiplied with elementary line-length (i.e. d) gives the total length of the boundary. Since conservative and dissipative energies are expressed in terms of horizontal and vertical lengths, respectively, the line elements can be further specified accordingly as $N_{\|}$and $N_{\perp}$. The NumberLines is defined as, NumberLines $=1 / d$. The conservative and dissipative energies can thus be expressed in terms of $N_{\|}$and $N_{\perp}$, i.e. they can be defined in terms of appropriate states. The shapes thus can be described by ratios, sums, and differences of dimensionless numbers.

The entropy of discrete systems so-called the Shannon entropy can be calculated from the number of probable states. The change occurs along the vertical direction and the number of new states is generated along this direction as seen in Figure 10.

This type of surface is studied in the literature in relation to crystal growth. The Kossel-Jackson-Temkin models of crystal growth are quite often referred to in the literature. The Kossel-Temkin entropy introduces a length scale into evolving thermodynamics of diffuse interfaces [9] [28] [29] [30]. The entropy of the crystal boundary can be simply calculated from the already formed surface where every step corresponds to a new state.

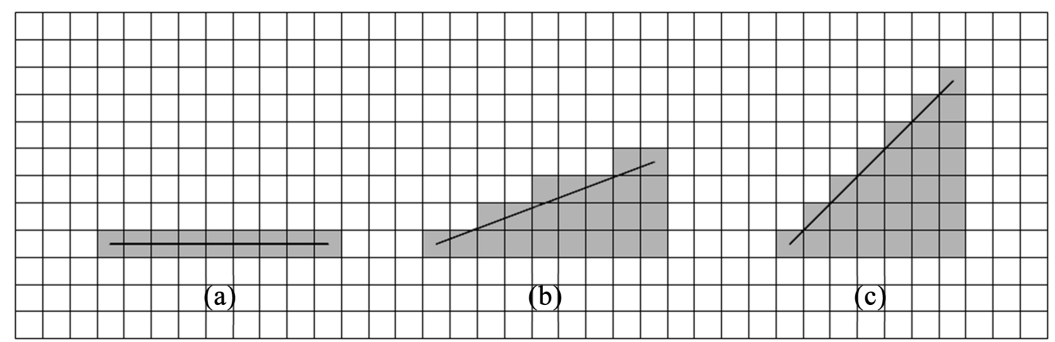

Figure 10. Entropy states in a discrete system. 
In Figure 10(a) there is a single surface (i.e. one state), while there are four surfaces (i.e. four steps or four states) in Figure 10(b), and there are seven states in Figure 10(c). As we go up along the vertical direction new surfaces are generated depending on the slope. Note that the number of states is equal to the number of cells along the vertical direction in all cases in Figure 10. Such a discrete geometry was revealed in many systems in the literature. For instance, the entropy of the distribution of notes in a song also exhibits a surface similar to the surface of a crystal, and its evolving entropy can be calculated by using the Shannon entropy formula [31].

The Shannon entropy is defined as the logarithm of states. When the cell size " $d$ " (see Figure 2) is decreased we get a greater number of states. When $d \rightarrow 0$, we can take the natural logarithm of the vertical length to calculate entropy, i.e. we use the Boltzmann entropy if the system is not discrete. Now, we can write,

$$
\begin{gathered}
\text { entropy } \sim \log (\# \text { of states }) \\
\text { \# of states } \sim \text { vertical height } \sim \ell_{\perp}
\end{gathered}
$$

When $\ell_{\perp}=1$ we have $\log (1)=0$, and this is the case for Figure 10(a). Entropy can be related to length $\ell$ and slope $m$ as follows.

$$
\begin{gathered}
\text { slope }=m=\tan \theta=\frac{\text { vertical lenght }}{\text { horizontal length }}=\frac{\ell_{\perp}}{\ell_{\|}} \\
\ell_{\perp}=m \ell_{\|} \\
\ell^{2}=\ell_{\perp}^{2}+\ell_{\|}^{2}
\end{gathered}
$$

From Equations (13) and (14) one gets,

$$
\ell_{\perp}=\ell \sqrt{\frac{m^{2}}{1+m^{2}}}
$$

Therefore,

$$
S \sim \ln \left(\ell_{\perp}\right)=\ln \left(\ell \sqrt{\frac{m^{2}}{1+m^{2}}}\right)
$$

The entropy of an edge can be expressed either in terms of the vertical component of length $\left(\ell_{\perp}\right)$ or in terms of the length $(\ell)$ and the slope $(m)$.

\subsection{Entropy and Shape Temperature of Edges of Triangles}

When $\theta=0^{\circ}$ for one of the edges like Figure 3(a) its heat content is zero, and the associated temperature change also comes out to be zero, (i.e. $\Delta T$ ). We can take the temperature of a horizontal edge to be zero as ground state temperature. Entropy and temperature both increase with the increase of $q$, or with the increase of $\ell_{\perp}$.

Equation (16) can be used to calculate the edge entropy of any polygon, and the total entropy can be simply found out from the sum of the entropies of all edges, such that, 


$$
\Delta S \sim \sum_{1}^{n} \ln \left(\ell_{i, \perp}\right)
$$

Note that we omitted the constant in Equations (16) \& (17). The change of $\Delta S$ for triangles is shown in Figure 11.

The total edge entropy of the triangle shows a peak at $45^{\circ}$ in all cases except in the right isosceles triangle. This might be because maximum randomness occurs at $45^{\circ}$, and we also have a maximum $q$ value as seen in Figure 8. Although the maximum $q$ of the right isosceles triangle also shows up at $45^{\circ}$, its entropy is minimum at $45^{\circ}$ as seen in Figure 11 . So, it is not only the length but also the angle between two adjacent edges which affect entropy. In fact, in Figure 8 the shapes of curves of a right triangle and right isosceles triangle also differ extensively from each other. The decreasing order of total entropy (i.e. the area covered under the curve) goes as, isosceles, right isosceles, obtuse, scalene, and equilateral. The isosceles has the highest while the equilateral has the minimum entropy.

The temperature associated with an edge can now be easily found out from the thermodynamic relation,

$$
T_{s} \sim \frac{q}{\Delta S}
$$

It should be kept in mind that this is not the kinetic temperature connected to $(1 / 2) m v^{2}=(3 / 2) m v^{2} k T$. Anyway, Equation (18) is a general expression to relate heat, entropy, and temperature. It is more appropriate to talk about "temperature" like attribute rather than kinetic temperature, or better to say "shape temperature", $T_{s}$. The " $\log _{2}$ " expression used in Shannon entropy need to be converted to "ln" in Equation (18). However, since Equation (18) is expressed as a relation we may not need to do it.
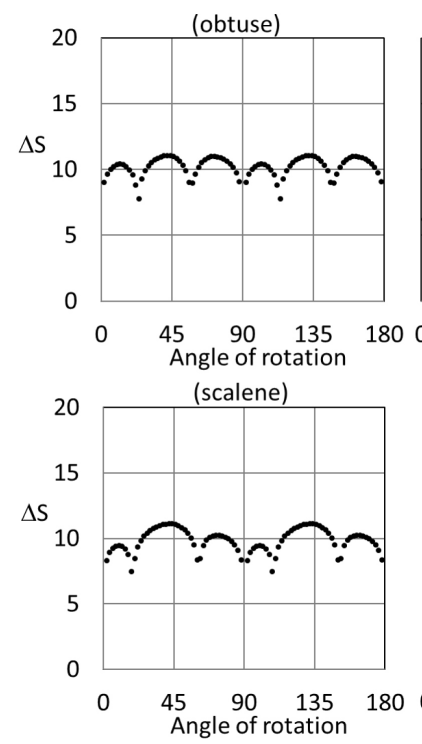

Figure 11. Change of entropy of triangles with $\theta$.
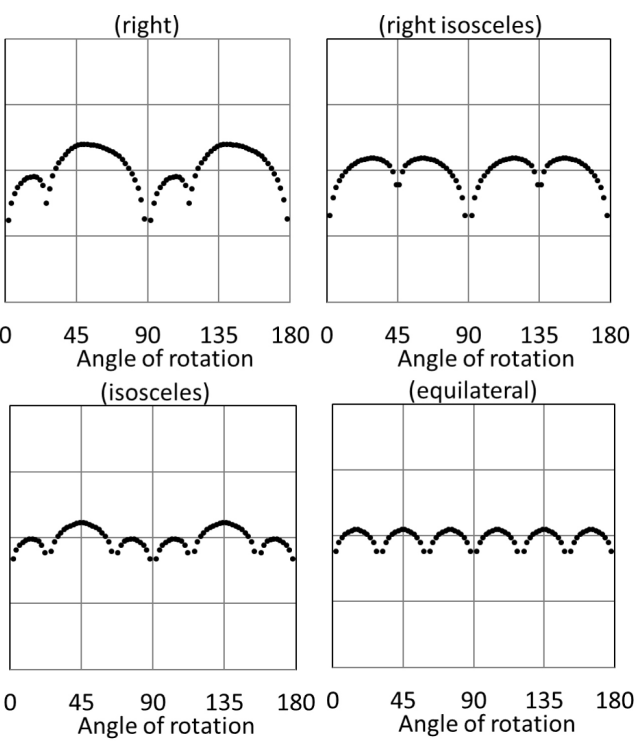

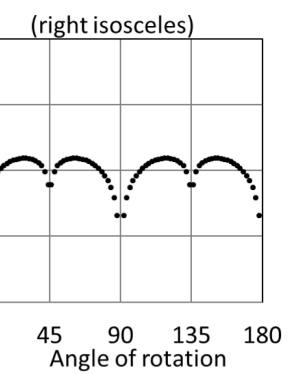


The change of shape temperature of each edge with the angle of rotation was calculated for each triangle, and it was found out that the general trends of the patterns were similar. Therefore, the changes of $\Delta S$ and $\theta$ were given only for scalene in Figure 12 as an example. The magnitudes are connected to the lengths only as the physical terms like heat capacity and ideal gas constant are all taken to be unity.

The lengths of $\mathrm{AB}, \mathrm{BC}$, and $\mathrm{CA}$ are $80,102.96$, and 94.87 units, and the angles $\hat{\mathrm{A}}, \hat{\mathrm{B}}$, and $\hat{\mathrm{C}}$ are $71.57^{\circ}, 54.46^{\circ}$, and $53.97^{\circ}$ respectively. The magnitudes of maximum $T_{s}$ values of $\mathrm{AB}$ and $\mathrm{BC}$ were close to each other, but that of CA was significantly high. It should be related to the high value of $\hat{A}$, i.e. the angle that the edge CA makes to the base. The patterns of $\Delta S$ and $T_{s}$ cope with each other for $\mathrm{AB}$ as they are both equal to zero at $\theta=0^{\circ}$, but they differ for $\mathrm{BC}$ and $\mathrm{CA}$.

\subsection{Entropy and Shape Temperature of Edges of Higher Polygons}

Entropy was calculated by using Equation (17) as in the former case. The patterns obtained are shown in Figure 13. They came out to be in perfect symmetry in every quarter, thus only $0^{\circ}-90^{\circ}$ interval was used in Figure 13.
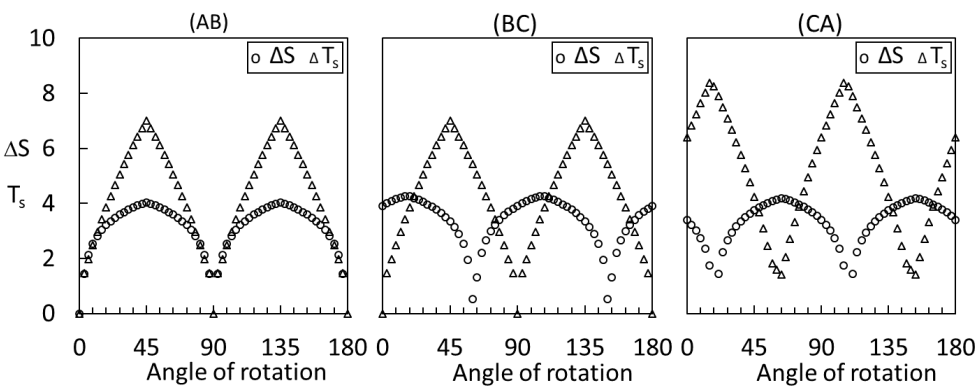

Figure 12. Change of entropy and shape temperature of a scalene triangle with $\theta$. $\mathrm{AB}, \mathrm{BC}$, and $\mathrm{CA}$ denote the edges, respectively, $\mathrm{AB}$ being the base.

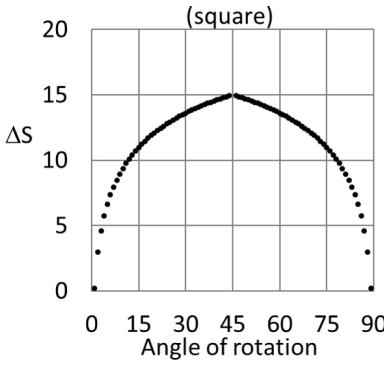

(hexagon)

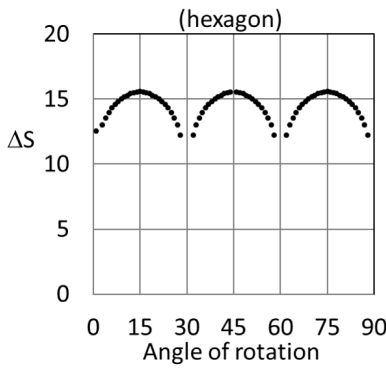

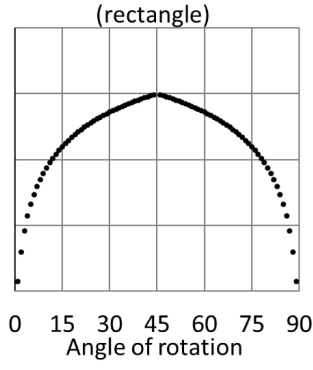

(heptagon)

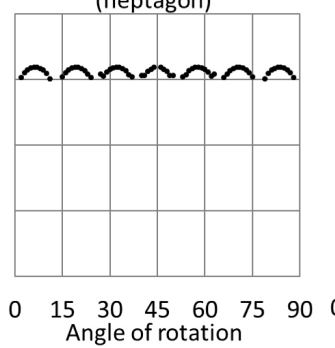

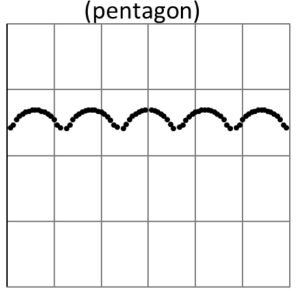

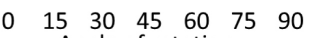

Angle of rotation

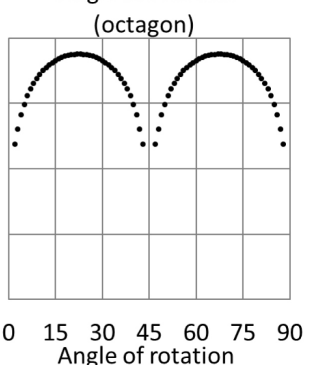

Figure 13. Change of entropy of higher polygons with $\theta$. 
The entropies of the square and rectangle came out to be equal being independent of the ratio of edges of the rectangle as long as the area is kept constant. The amplitude of repeating patterns follows similar behavior to that of $q$ shown Figure 9; heptagon has the lowest amplitude change, then comes pentagon, hexagon, octagon, and square.

The order of increase of total entropy that includes all the edges of the polygon is as, square, pentagon, hexagon, heptagon, and octagon. The entropy increases with the increase of the number of edges, and in the limit, we came up with a circle that will have the maximum surface entropy with a smooth surface having no amplitude fluctuations. It has the highest surface entropy despite it has the lowest surface energy (see Section 3.2). Therefore, it has the lowest shape temperature according to Equation (18). The increase of total entropy with the increase of the number of edges in polygons can be understood by simply comparing square and octagon, the former is four-sided and the latter eight-sided. Octagon is obtained from the square by fragmenting each side of the square into two, and the fragmentation process increases entropy, conceptually. As mentioned in the Introduction, the number of edges in Euler's topological rule corresponds to the number of components in Gibb's phase rule. The increase of the number of components in a system increases the Boltzmann entropy, and correspondingly the increase of the number of edges increases the entropy of polygons.

The change of entropies and shape temperatures with the change of $\theta$ for the base edge of higher polygons are shown in Figure 14. As it is seen the magnitude of temperature decreases as the number of edges increases from square to octagon. It theoretically reaches its minimum when a circle with the same area of polygons is achieved finally.
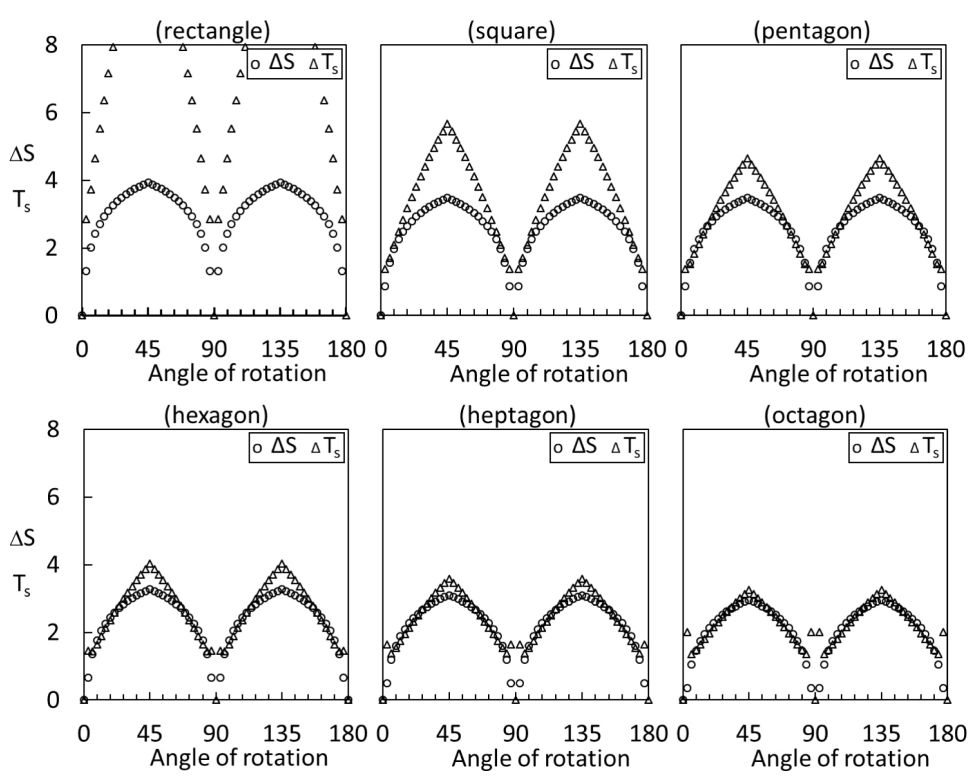

Figure 14. Change of entropy and shape temperature of the base edge AB of higher polygons. 
The base edge of the rectangle is larger than that of the square, and both the entropy and the temperature of the base $\mathrm{AB}$ came out to be larger than that of the square as seen from the first and second graphs of Figure 14.

In classical thermodynamics, "nothingness" is defined by zero degrees Kelvin $(K)$ state where there is no energy, no heat, no entropy, no temperature. It is the very ground state which cannot be achieved by physical means. When do we get this state in geometry? It is the "point" that has no length, no energy, no entropy, and no temperature; no matter how we rotate it we cannot assign any physical attribute to it. The definition of a point from the times of Aristotle and Euclid to our age never changed; it is a primitive (or a apiori) notion upon which we build the geometry just like we build temperature and thermodynamic energy on zero degrees Kelvin. Zero degrees Kelvin cannot be achieved by decreasing the temperature no matter what is physically done; similarly, "point" cannot be reached no matter how much we contract a line.

In polygons the lengths of sides are constant, i.e. the shape is constant, but it takes a different appearance as we rotate the polygon. As mentioned before, Birkhoff's [17] claim of "rhombus is less aesthetic than square" can be interpreted in terms of entropy concept instead of his "non-pleasing parameter" which is a non-physical concept. As we rotate the square by $45^{\circ}$ its entropy goes up to maximum when $\theta=45^{\circ}$, as seen in Figure 14. All of its attributes look more or less like that of a rhombus. In addition, the temperature also goes up to the maximum as we go from square to $45^{\circ}$ rotated square. There is an intimate relation between aesthetics and entropy [31] [32] [33].

\section{Thermodynamic Attributes of Curves}

In polygons the edge lengths are constant, and the calculations for energy and entropy are done for each edge at varying angles, and then they are summed up to find out the total value as described before. In the case of curves, first, we need to specify the beginning and the end of the curve. Then, we need to find out the length of our vector extending from an origin to a specific point on the curve.

In the calculations carried out the curves were not rotated but the varying distance from the origin to any point on the curve was taken as the varying parameter. The specific curves studied are circle, sine curve, spiral, and exponential. Among these, the circle was considered first as it has constant vector length from the origin (i.e. radius), but the length changes with $\theta$ in the other curves. The diagrams of these curves are given in Appendix B.

The total and dissipative energies are calculated from Equation (8), and Equation (9), respectively. The calculation of entropy change in curves needs a bit of attention. Let us consider a decaying exponential curve given in Figure B1. In all entropy calculations that we did for polygons, we took the base edge as our reference and changed the angle by $1^{\circ}$, where the length was constant. A decreasing exponential curve never crosses the horizontal axis, and the smaller the angle $\theta$ the bigger the length of our vector from the origin, meanwhile, the smaller the 
vertical component of length (i.e. $\ell_{\perp}$ ). As $\ell_{\perp}$ gets smaller than " 1 " the logarithmic equation gives negative values for entropy. Theoretically, there is no problem with negative entropy values, because, one may get negative entropies according to Equation (17) as one may use any arbitrary length which is less than 1 , (i.e. $\ell_{\perp}<1$ ). The critical question here is to what specific point we must refer to, to calculate the entropy difference. The $\Delta S$ goes to zero when $\theta=0^{\circ}$, because, $\ell_{\perp}=0$ at $\theta=0^{\circ}$. Therefore, for $\theta<1^{\circ}$ angles we need to make an extrapolation to set the minimum entropy state. For any curve, this can be done by considering three points on the curve having angles closest to $\theta=1^{\circ}$ but smaller than $1^{\circ}$, and then make extrapolation to calculate the corresponding entropy to $\theta=0^{\circ}$ state. It gives us the minimum entropy value. For instance, as the small circle is closer to the origin than the big circle (see Figure B1), we take the smallest three angles of the small circle close to the horizontal axis and make the extrapolation accordingly. Since the " $d$ " value must be the same, we use this value also for the bigger circle. Then the difference between this value and the entropy value calculated at any angle (i.e. $\log \ell_{\perp}$ ) gives us $\Delta S$. We did not have this issue in polygons, because, we had $\log \ell_{\perp}>1$ all the time, and also, we had constant edge lengths. The minimum entropy of any edge when $\theta=0^{\circ}$ was zero.

In polygons, we simply had $\ell_{\perp} \sim \ell \sin (\theta)$, but in curves we had $\ell_{\perp} \sim \ell(\theta) \sin (\theta)$ no matter whether $\ell$ changes with $\theta$ or not, and the entropy change in a circle is found out by the same procedure.

After finding the change of $\Delta S$ with $\theta$ the shape temperature can then be calculated using Equation (18).

\subsection{Circle}

The arc of the first quadrant of the circle was considered only, because, there is a perfect symmetry in the other quadrants. As seen from Figure 15 both $E$ and $q$ increased with the increase of length (i.e. $\ell$ or radius $r$ ) as expected. The $\Delta S$ values of the big circle came out to be larger, because, its $\ell_{\perp}$ values are bigger than that of the small circle. Their $T_{s}$ values also follow the same pattern. As seen from Figure 15 the maximum occurs at $\theta=45^{\circ}$ in all cases as expected.

\subsection{Sine Curve}

The calculations for sine curves were carried out in the section shown in Figure B1, where the arrow indicates a representative case. The coordinates used for calculations were located at the middle of the half-wave such that the maximum height occurs on the vertical axis as in the case of a circle. In Figure 16 the symbol A in the legend refers to the amplitude of sine wave, and the behaviors at three different amplitudes were studied; these are $0.5,1$, and 2 , respectively. The maximum total energy occurs at different angles depending on the value of amplitude, and the associated $\theta$ value increases with the increase of amplitude. In the case of dissipative energy, the maximum occurs at $\theta=45^{\circ}$ in all cases. The 

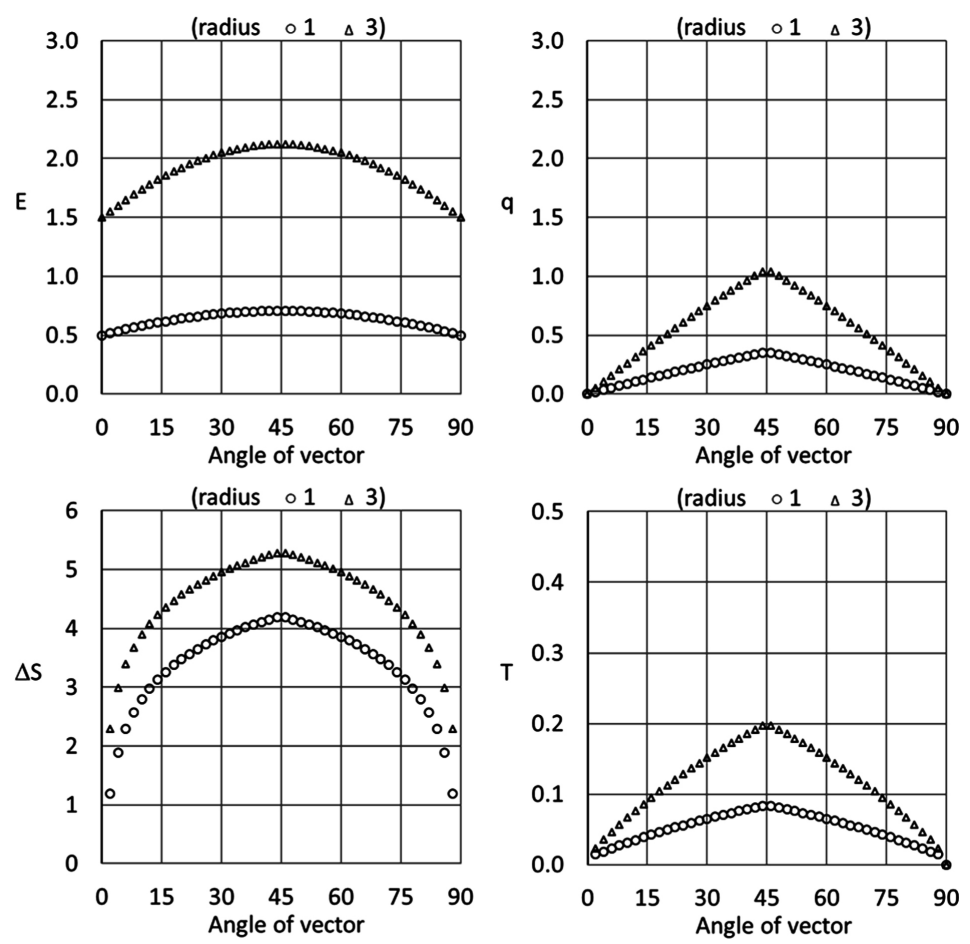

Figure 15. Total energy, dissipative energy, entropy, and temperature of circles.
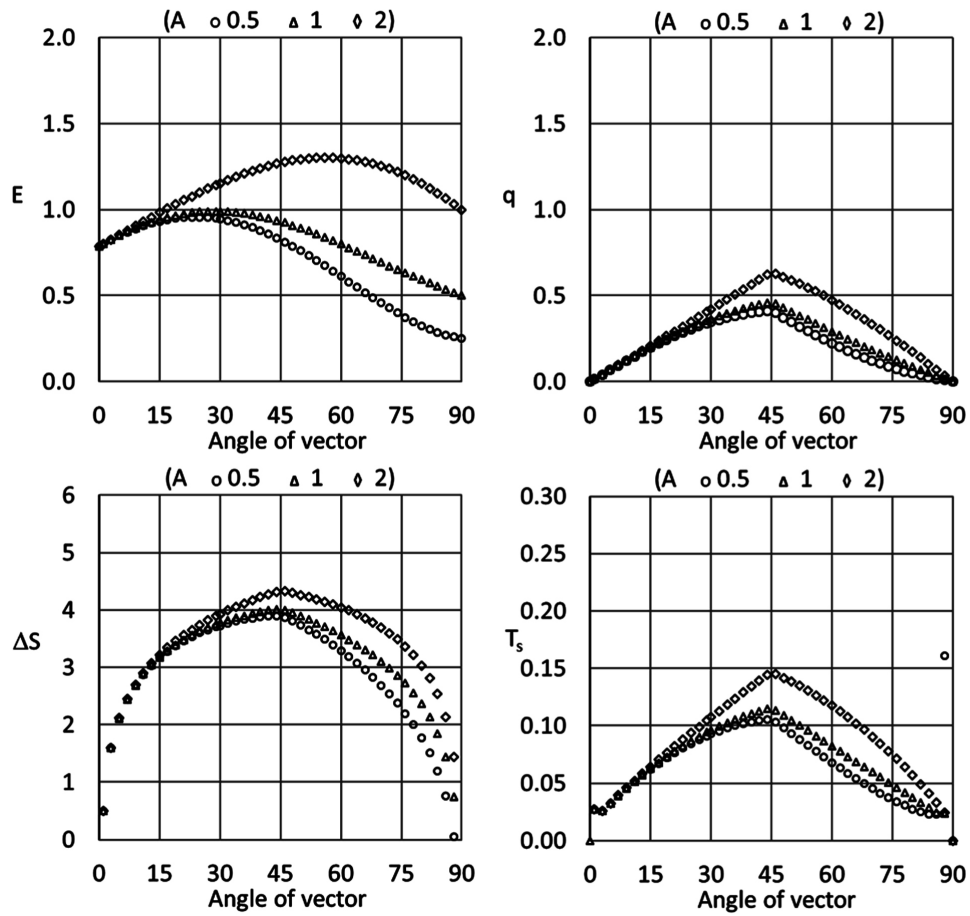

Figure 16. Total energy, dissipative energy, entropy, and shape temperature of sine curves.

effect of the vector angle becomes more distinctly pronounced between $45^{\circ}$ $90^{\circ}$, the curves distinctly separate in this region. It is nothing but the shape effect of a sine curve. The same effect is also observed in $\Delta S$ and $T_{s}$ curves. In all cases 
larger amplitudes resulted in larger changes for all terms studied, i.e. in $E, q, \Delta S$, and $T_{s}$.

In these two cases (i.e. circle and sine curve) the effect of the change of amplitudes was taken into consideration. The effect of the coefficient of the variable will be demonstrated below for two other different cases, one for spiral and the other for exponential curve, where the amplitudes were kept constant. In a spiral, the expansion of the curve and thus the curvature depends directly on $\theta$. In an exponential curve, curvature depends on the variable of the equation (i.e. $x$ in $y \sim \exp (-k x)$. The change of $\theta$ of a vector thus depends on the value of $\exp (-k x)$, and actually on the magnitude of the constant $k$.

\subsection{Spiral}

Two different spirals at different rates of expansion were studied (see Figure B1). The fast-expanding one results in larger total energy, dissipative energy, entropy change, and temperature as seen from Figure 17. For the entropy case, a magnified view of the upper part of one of the peaks was also displayed as seen in Figure 17. The angle was changed between $0^{\circ}-720^{\circ}$, and the amplitudes of oscillating waves continue to increase in all cases. The increase of $T_{s}$ with the increase of expansion rate, (i.e. the " $b$ " constant in the equation, $y=a \exp (b x)$ agrees with the globule-to-helix transition in some macromolecules. The increase of temperature or the use of some solvents transforms a macromolecule from globule into helical form. The globule state is a much more compact form and has smaller entropy whereas helix has larger entropy. In Figure 17 the one with a smaller " $b$ " value has a more compact structure than the one with larger " $b$ " value. The former has smaller entropy than the latter.
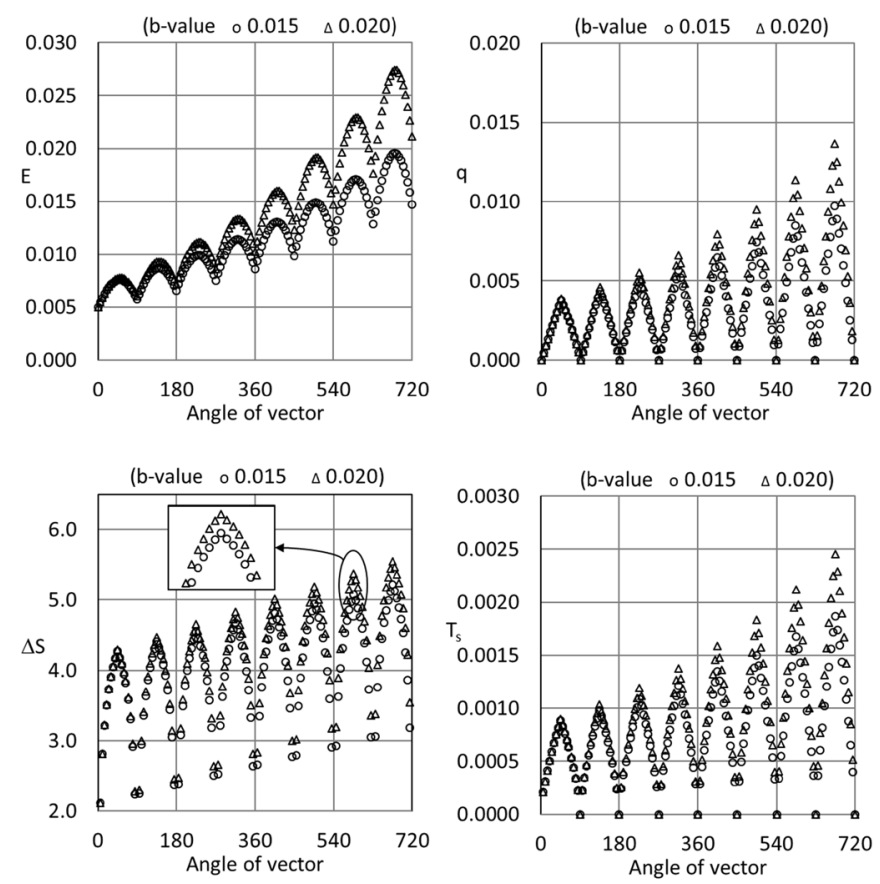

Figure 17. Total energy, dissipative energy, entropy, and temperature of spirals. 


\subsection{Exponential Curve}

In this section, two exponential curves with different decaying rates were considered. The one having a higher rate constant (i.e. $k=1.5$ case) decays faster than the one having a smaller rate constant (i.e. $k=1.0$ case), (see Figure B1). Therefore, the vector length from the origin to any point on the fast decaying curve comes out to be smaller than the corresponding vector extending to the relatively slow decaying curve (i.e. $k=1.5$ case). As a result, the total and dissipative energies of the fast decaying curve came out to be smaller than those of slowly decaying curve as seen in Figure 18.

This result is true also for entropy change. The dissipative energies reached their maximum in both cases at $\theta=45^{\circ}$, but the temperature does not quite follow this trend. For $k=1.0$ case temperature reaches its maximum at $\theta=45^{\circ}$, but for $k=1.5$ case, it reaches its maximum at around $60^{\circ}$. After this angle, the $T_{s}$ of the fast decaying curve (i.e. $k=1.5$ case) goes over the slow decaying curve (i.e. $k=1.0$ case).

From all these four cases (i.e. circle, sine curve, spiral, and exponential curve) it can be concluded that any parameter which affects the angle of the vector drawn from an origin to the curve changes the physical attributes, i.e. total energy, dissipative energy, entropy, and temperature, or in general the states concerning these attributes. The parametric changes are essentially dependent on the amplitude and the coefficient of $\theta$ angle.
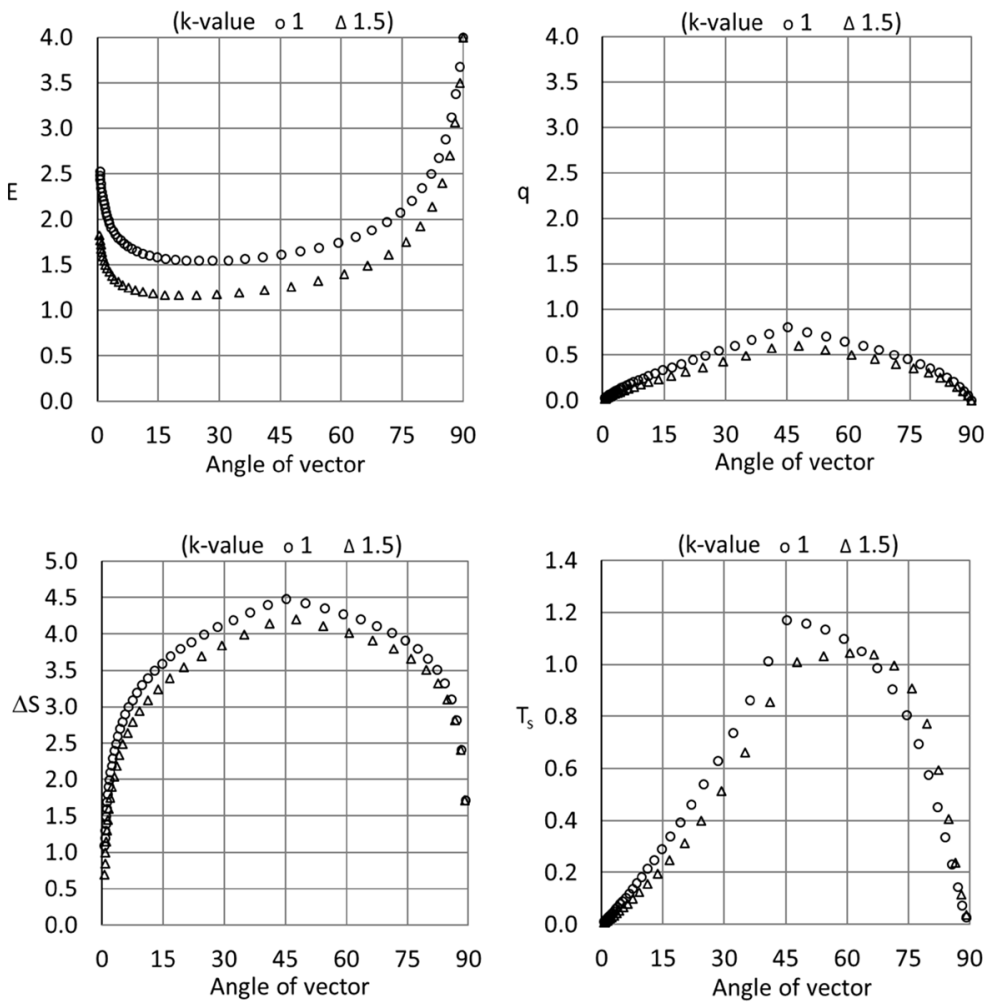

Figure 18. Total energy, dissipative energy, entropy, and temperature of exponential curves. 


\section{Time-Dependent Cases}

The entropic behaviors of exponentially decaying curves reveal some interesting implications. If the " $x$ " variable of the exponential curve is substituted by time " $t$ " parameter, then we can discuss the entropy change on the grounds of the time domain. Fast or slow decays refer to velocities of rates. For instance, the decay rate of a chemical compound " $A$ " with an initial concentration of $A_{0}$ into anything can be simply expressed as,

$$
\begin{aligned}
& A \rightarrow \text { anything } \\
& \frac{\mathrm{d} A}{\mathrm{~d} t}=-k A
\end{aligned}
$$

This is known as a first-order irreversible chemical reaction in chemical kinetics. Its integration gives,

$$
A=A_{0} \mathrm{e}^{-k t}
$$

Now we can elucidate it in terms of the entropy concept. We know that $\exp (i \omega t)=\cos (\omega t)+i \sin (\omega t)$, where $\omega$ denotes frequency. Thus, a change in $\omega$ corresponds to a change in $k$, and the entropy of a time-dependent trigonometric function becomes dependent on its frequency.

In Figure 19 the comparison of two sine curves was given. The $\lambda / 2$ denotes the numerical value of half of the wavelength. The amplitude was taken to be $A$ $=1$ in both cases. The triangular symbol refers to the case shown by the same symbol in Figure 16. The sine curve designated by the square symbol refers to
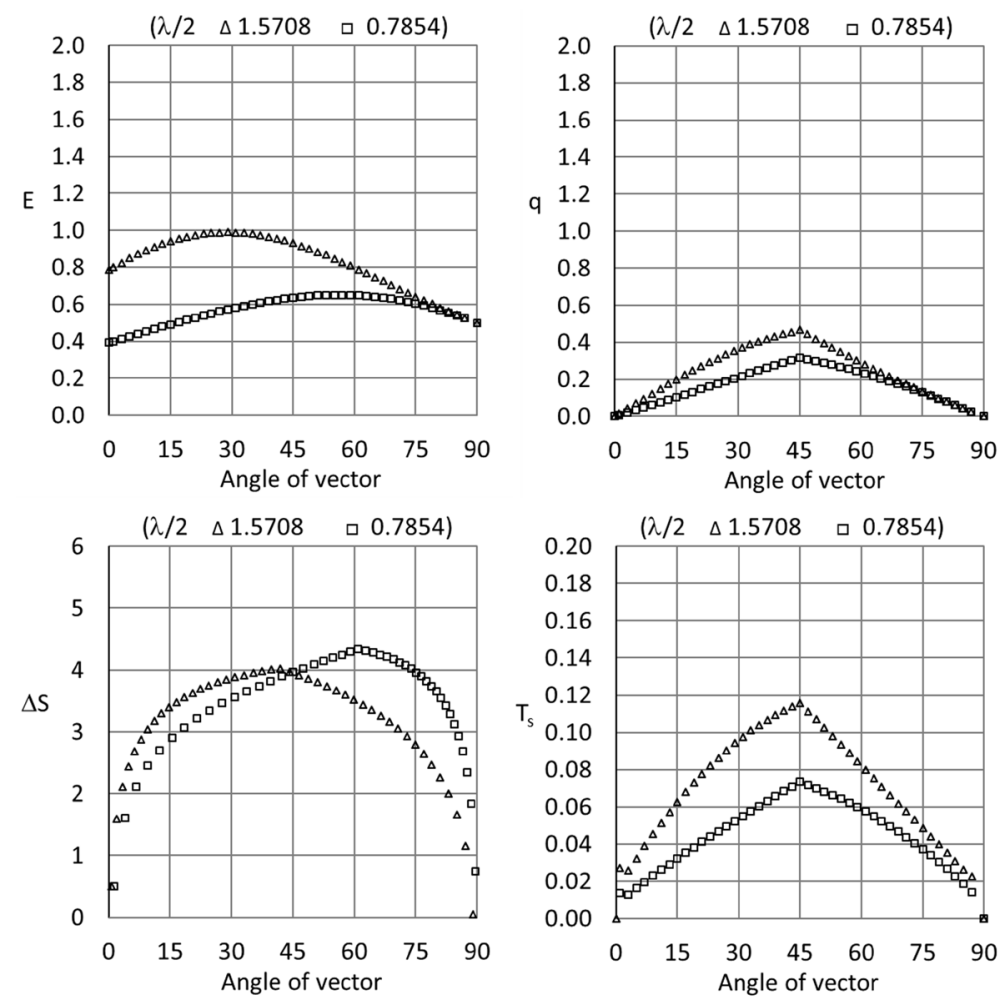

Figure 19. Behaviors of sine curves at different frequencies. 
the case where the frequency is increased twice. When the frequency is doubled the total energy and dissipative energies decrease as the length of the vector decreases. As seen from Figure $19 \Delta S$ of the high-frequency case (i.e. 0.7854 with square symbol) goes over the low-frequency case after $\theta=45^{\circ}$. The behavior of $T_{s}$ is like the behaviors of $E$ and $q$. However, in Figure 18 the $T_{s}$ of the fast decaying curve goes over the slow decaying curve after $\theta \simeq 60^{\circ}$.

\section{Curvature}

Curvature appears in thermodynamics in relation to surface properties. For instance, in a curved liquid-vapor interface the change of vapor pressure is given by the Kelvin equation,

$$
\ln \frac{p}{p_{\text {sat }}}=\frac{2 \gamma V_{\mathrm{m}}}{R_{\mathrm{gas}} T} \frac{1}{r}
$$

where $p$ is actual pressure, $p_{\text {sat }}$ is saturation pressure for the flat surface, $\gamma$ is the surface tension of the droplet, $V_{\mathrm{m}}$ is molar volume, $R_{\mathrm{gas}}$ is the universal gas constant, and $T$ is temperature. The change of vapor pressure or chemical potential (i.e. $\mu$ ) across a curved surface is known as the Gibbs-Thomson effect, and the change in Gibbs free energy or chemical potential is given by [27],

$$
\Delta G=\frac{2 \gamma V_{m}}{R_{\text {gas }}} \frac{1}{r} \sim \mu
$$

These two equations are related to each other only by the temperature term. Curvature reveals in both pressure change and interfacial energy change. In other words, curvature affects both pressure and interfacial energy. Therefore, curvature directly affects the thermodynamic properties and it has a predominant role in the formation of shapes [34]. Curvature, entropy, and shape were discussed within the same framework in the past [11] [12]. As mentioned in the Introduction, "dissipative reactions or transformations give rise to curvature and thus a thermodynamic length" and also "dissipative reactions or changes give rise to curvature" [5]. The dissipative energy $q$ and the associated $\Delta S$ and $T_{s}$ terms may be thought to be connected to curvature. Temperature is an intensive property in thermodynamics, and curvature also is an intensive property in geometry. We may check if there is a connection between curvature and $q, \Delta S$, and $T_{s}$.

In Figure 20 there are two vectors $\mathrm{AC}$ and $\mathrm{A} \mathrm{C}^{\prime}$ which are of equal length. $\mathrm{AC}$ resembles vector (c) in Figure 3, and A C'resembles vector (b). Now let us consider the mirror images of $\mathrm{AC}$ and $\mathrm{A} \mathrm{C}^{\prime}$ which are $\mathrm{BC}$ and $\mathrm{B} \mathrm{C}^{\prime}$, respectively.

As the height $\mathrm{CD}$ contracts to $\mathrm{C} D$ 'the dissipative component of energy (i.e. q) decreased. Meanwhile, $\mathrm{AD}$ extends to $\mathrm{A} \mathrm{D}^{\prime}$ 'and so the conservative component gets larger. In other words, randomness decreases as CD decreases in magnitude, because, the number of broken bonds on the edges decreases. Thus, the contraction of $C D$ to $C D$ 'has two effects, one is the decrease of dissipative energy and the second is the decrease of entropy. 


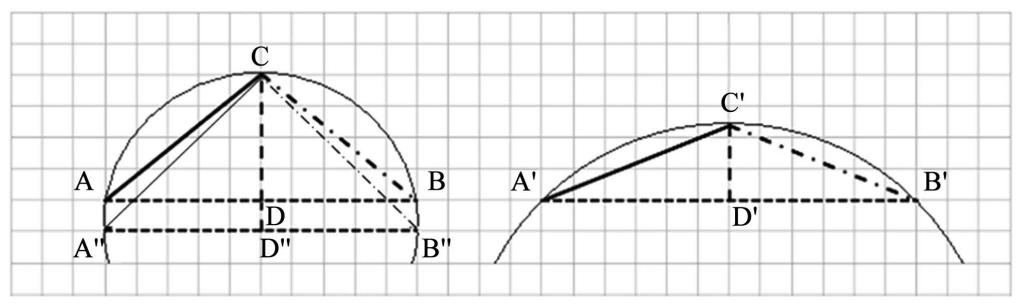

(a)

(b)

Figure 20. Curvature.

Let us draw a circle passing through A, B, C, and also through A', B', and C.' The magnitude of the radius of circle depends both on the length of vector (i.e. AC or A C) and its angle. Therefore, the circle radius $(R)$ or the curvature $(\kappa=1 / R)$ carries information about the value of $q$ and $\Delta S$. Curvature appears both in dissipative energy and entropy just like temperature term in thermodynamics. If $\mathrm{CD}\left(\right.$ or $\left.\mathrm{C}^{\prime} \mathrm{D}^{\prime}\right) \rightarrow 0$, then $R \rightarrow \infty$ or $\kappa \rightarrow 0$, it refers to a flat smooth surface as in Figure 3(a) or Figure 7(a). Therefore, curvature seems to be a good candidate to be used in dissipative properties. However, it is not quite true on a quantitative basis, because the curvature is defined in terms of geometric radius and we are confined to the height $\mathrm{CD}$ (or CD) in Equations (9) \& (17), not to the radius. The radius of curvature extends further away beyond point $\mathrm{D}$ (or D), but the calculations of $q, \Delta S$, and $T_{s}$ are all based on CD (or CD) provided $\theta \leq 45^{\circ}$. There is only one special situation that curvature can exactly cope with $\mathrm{CD}$. This is the case when we have $\mathrm{AD}=\mathrm{DC}$, that is when $\mathrm{AB}$ is equal to diameter, so that $\mathrm{CD}$ is equal to the radius $R$ in circle. This is the case shown by the triangle $\mathrm{A}^{\prime \prime} \mathrm{B}^{\prime \prime} \mathrm{C}$ in Figure $20(\mathrm{a})$, where $\mathrm{A}^{\prime \prime} \mathrm{D}^{\prime \prime}=\mathrm{D}^{\prime \prime} \mathrm{C}$, and $\theta=45^{\circ}$.

The study carried out on planar objects in this work can be extended to three-dimensional objects where calculations may be more cumbersome depending on the geometry of the shape. The increase in the number of symmetry axes of the object may provide relatively easier calculations. For instance, the study of a sphere that has perfect symmetry in all directions can easily be done. The smallest discrete distance " $d$ " then turns out to be the smallest discrete surface area in three-dimensional objects.

As mentioned at the very beginning, the viscoelastic and thermodynamic attributes of the shapes of polygons and curves were investigated in this work. In dynamic systems, for instance, in time series systems, it is not the finite trigonometric shapes but rather patterns form in time. The conservative and dissipative energies and the configurational entropies in such systems were discussed in the literature [35] [36] [37] [38]. Although there are similar approaches to evaluate the properties, in both cases their scientific grounds are different.

\section{Black Hole Temperature}

In black hole physics, the attributes were established mainly by making analogies with other physical laws. For instance, the ever-increasing surface area of the black hole due to material suction from the surrounding space or due to some 
other processes such as merger black holes was thought to mimic the second law of thermodynamics. Christodoulou proved that the irreducible mass of a black hole is proportional to the square root of the surface area [39]. Bekenstein interpreted the irreversible increase of the surface area of a black hole as black hole entropy [40], and Hawking confirmed Bekenstein's conjecture and fixed the proportionality constant using thermodynamic concepts [41] [42]. The entropy equation is given by,

$$
S_{b h}=\frac{k}{4} \frac{A}{\ell_{P}^{2}}
$$

where $k$ is the Boltzmann constant and $\ell_{P}$ denotes the Planck length, $\ell_{P}=\sqrt{G \hbar / c^{3}}$ where $G$ is the gravity constant. The first law of thermodynamics of Schwarzschild black hole was set to be,

$$
\delta M=\frac{\kappa}{8 \pi} \delta A
$$

where the units are such that $k=G=c=1$. $M$ denotes the mass and $\kappa$ denotes the surface gravity which is the gravitational acceleration experienced at the surface. It was proposed that $\kappa / 8 \pi$ is analogous to temperature in the same manner $A$ is analogous to entropy [41]. The following relations were proposed to describe the thermodynamics of black holes [43] [44] [45] [46].

$$
\begin{gathered}
S_{b h} \sim A \sim M^{2} \\
\kappa \sim \frac{1}{M} \\
T_{b h} \sim \frac{h}{M}
\end{gathered}
$$

The last formula implies that the Schwarzschild black hole is black (i.e. $T_{b h}=$ 0 ) in the classical limit, i.e. $h \rightarrow 0$.

Hawking conjectured that virtual particles can form as the pair of particle and anti-particle just outside the event horizon due to quantum effects. Each pair forms out of nothing with zero total energy at positive and negative energy states. It is possible before annihilation that one of them may fall into a black hole and the other runs away as Hawking radiation. As the one with negative energy is swallowed by the black hole it takes away energy from the back hole, i.e. reduces its mass. In time, a black hole is supposed to evaporate and diminish. The temperature of the black hole increases as it gets smaller due to its evaporation as implied by Equation (27) [43].

The black hole entropy and temperature concept has been disputed for decades and it has not settled yet. First of all, all horizons possess entropy and temperature not necessarily black holes [47] [48] [49] [50]. Although it makes sense, the black hole entropy concept still is a shrouded concept for us [51]. The temperature concept of a black hole should not be taken and should not be used for emission processes in black holes [52]. Helfer commented that "none of the derivations that have been given of the prediction of radiation from black holes is 
convincing" [53]. An important point is that horizons can be observer-dependent but entropy is an observer-independent attribute [54]. The related discussions expanded our knowledge and yielded new fundamental ideas such as holographic models [55] [56] [57], and also to interpret gravity as an emergent property like entropy such that gravity is a kind of entropic force [58] [59].

Despite all these objections, the existing definitions of black hole entropy and temperature keep surviving. The entropy defined by Equation (23) is reasonable, and it is similar to classical systems. Black hole entropy can be considered to be a contribution to the entropy content of the universe.

After this concise survey, we can now make a few comments on black hole temperature.

\subsection{Curvature and Temperature}

When the first law of thermodynamics was defined by Equation (24), $\kappa$ necessarily turns out to be a temperature term. However, $\kappa$ is surface gravity and has something to do with acceleration and so with the curvature of spacetime.

As mentioned above in Section 8 there is a kind of relation between curvature and entropy and thus between curvature and temperature, but this relation is not one-to-one. The heat and entropy and thus temperature all are determined from the vertical axis by using the length of $C D$ or $C D^{\prime}$ (Figure 20). However, the curvature cannot be found out by using $\mathrm{CD}$ or $\mathrm{CD}$. Only and only in the special case when the vertical length is equal to the radius, i.e. when radius = $C^{\prime} \mathrm{D}^{\prime \prime}$ (Figure 20) we have a one-to-one correspondence between curvature and entropy and thus temperature. Therefore, it is not correct to relate $\kappa$ directly to temperature, because, it is associated with acceleration and thus with curvature, but the temperature is not related to curvature. Only in spherical geometry like that of the black hole we have the equality of radius and height to calculate entropy and temperature for an outside observer. If we think of a hypothetical black hole in the shape of an ellipsoid then we would have varying $\kappa$ and thus varying temperature profile on the surface leading to instability. It is better to comprehend $\kappa$ as a term that influences the temperature.

As explained in the former sections, the surface entropy and temperature of geometric shapes like polygons or curves can be calculated with reference to background geometry as seen in Figure 1. Although there are some proposals about the inner structure of black holes the general understanding is that it probably has a uniform structure like empty space. The empty space is not empty as we know from quantum electrodynamics and quantum chromodynamics. We may conjecture that the inner structure of black holes may have a kind of discrete structure as quantum gravity implies it. In fact, according to hologram theory or topological quantum theory, all inner physical attributes can be projected onto the boundary [55], and black hole entropy (or area) involves Planck's constant as seen from Equation (23). Let us think arbitrarily for a while of its discrete form to be like that of Figure 1. For an observer at the center, the 
change of the angle of rotation of a line (i.e. the radius line) connecting the center to any point on the circumference (see Figure 15) reveals varying entropies. This is the entropy with respect to the background (see Figure 3). For a bigger circle, the entropy increases in proportion to the length of radius whatever the angle is (Figure 15). For an observer outside the black hole, the inner structure is impermeable, and so the outside observer perceives the increase of entropy only with respect to the increase of radius, or surface area. This is so, because, the definition of entropy in physics is not made with respect to space, but rather on the probability distribution of the stuff in concern, and it reveals only at the surface in black holes.

In classical thermodynamics entropy has two components as in the well-known cylinder-piston expansion example, one is due to change in internal energy and the other is due to movement of the piston. The component associated with internal energy involves a temperature change while the one associated with the expansion (or contraction) of the piston involves the distance covered by the piston. If the expansion of the piston is isothermal, there is no change in temperature. The same is true also for an inflating balloon under isothermal conditions. The change in entropy only due to expansion of piston is given by,

$$
\Delta S=n R_{\text {gas }} \ln \left(\frac{V_{\text {final }}}{V_{\text {initial }}}\right)
$$

where $n$ is the number of moles, $R_{\text {gas }}$ is the gas constant, and $V$ is volume. The related energy change $(\Delta E)$ is,

$$
\Delta E=T \Delta S=n R_{\text {gas }} T \ln \left(\frac{V_{\text {final }}}{V_{\text {initial }}}\right)
$$

This description depends on the random collision of molecules where the ideal gas law ( $P V=n R_{\mathrm{gas}} T$ ) holds, $P$ indicating the pressure. Under very high pressures or very low temperature the ideal gas law does not hold, and a correction factor $Z$ is used to modify the gas law, such that, $P V=Z n R_{\text {gas }} T$. Under these severe conditions, the assumptions of ideality do not hold. For instance, at very low temperatures, molecules come close by to each other and start to attract each other. Without changing the temperature nonideality can be obtained also at very high pressures as the mean free paths between the molecules are extremely decreased, and the electrons of molecules start to influence each other's electronic states which result in again a kind of increase in chemical interaction between molecules. Under these conditions Equation (29) can be modified by introducing $Z$ such that,

$$
\Delta E=T \Delta S=Z n R_{\text {gas }} T \ln \left(\frac{V_{\text {final }}}{V_{\text {initial }}}\right)
$$

Shortly saying, the attractive forces enhance the matter interaction without changing the temperature. Since $n R_{\text {gas }}$ is constant one can interpret $Z$ to be a kind of factor that decreases $T$, because $Z<1$. Hence one can claim that "as at- 
traction increases temperature decreases", but this is a false claim, because, the temperature does need to be changed, and the increase in attraction is taken care of by $Z$. In other words, the effect of attraction is represented by $Z$ while $T$ is left unchanged.

In a cylinder-piston system, the entropy term connected to temperature change (i.e. associated with the change in internal energy) denotes thermal entropy, while the one connected to the motion (i.e. expansion of piston) denotes the configurational entropy. Thermal entropy involves temperature terms (i.e. $\left.\Delta S=n C_{\mathrm{v}} \ln \left(T_{\text {final }} / T_{\text {initial }}\right)\right)$ whereas configurational entropy does not (Equation (29)).

In black hole thermodynamics, the increase of surface area interpreted as the increase of entropy is connected to thermal entropy. The globule-to-helix transition in some macromolecules, and also the expansion of coil, or sphere, etc. may all be associated with the increase of temperature. The globule-to-helix transition can also be achieved by introducing a proper solvent into the medium. The solvent attracts the molecules of the globule such as the entangled linear chains of polymers and increases the end-to-end distance between chains. The "attraction force" between solvent and polymer chains functions as a kind of "increase of temperature" of the medium. Therefore, surface gravity $\kappa$ (Equation (24)) should not be connected to temperature but simply to the exerted attractive force revealed by gravity. $\kappa$ is the formal analogue of $Z$. If $\delta M$ increases in Equation (24) then $\delta A$ also increased but not in linear proportion, because, $\kappa$ also increases. The increase of $\kappa$ functions as the increase of $Z$ in Equation (30).

\subsection{Curvature and Evaporation}

Now let us consider Equation (22) which relates energy to curvature in classical thermodynamics. It simply means that the smaller the radius, or the larger the curvature the larger the surface energy. This equation applies to systems having a curved surface like a water droplet. The surface tension $\gamma$ represents the cohesive force or the extent of the attractive interaction of surface molecules. If we keep $T$ constant and decrease $r$ in Equation (21) then $p$ increases, and the evaporation from the surface is accelerated. The same conclusion is derived from Equation (22) such that if $r$ decreases the free energy change becomes larger, and the chemical potential $\mu$ becomes larger, therefore evaporation is enhanced. In Equation (22) the increase of curvature (e.g. 1/r) is reflected exactly as if the increase of temperature weakens or breaks down the bonds between liquid molecules enhancing the rate of evaporation. What changes is not the temperature but $\mu$. The same mechanism occurs in black holes. The loss occurring according to Hawking radiation decreases the radius and thus $\kappa$, and it then increases the rate of material loss; the smaller the $r$, the faster the rate of evaporation. The temperature need not be changed while the black hole is evaporating.

The description given in this section is quite fundamental and sets the framework without going into details. It can be elaborated in more detail by using the 
equations of general relativity and working with curvature tensor and energy tensor terms. It will be an entirely different subject to work on.

\section{Conclusions}

The total energy, dissipative energy, entropy, and temperature attributes of polygons and curves were demonstrated from the viewpoint of thermodynamic principles and viscoelastic theory. All these properties change as the polygon is rotated. The total energy, dissipative energy, entropy, and temperature depend on the edge lengths and the interior angles.

In regular polygons, the square has the highest and the octagon has the lowest total energy. The energy decreases as the number of edges increases implying that the circle has the lowest energy. However, total entropy increases with the increase of the number of edges, though, the edge length decreases as the area is kept constant in all cases. It implies that the circle has the maximum entropy even though it has minimum surface energy.

In curves, the total energy, dissipative energy, entropy, and temperature depend on the compactness of the curves. In other words, the amplitude and frequency in sine curves, amplitude, and decay constant in exponential curves, expansion constant in spirals play predominant roles in total energy, dissipative energy, entropy, and temperature.

In black hole thermodynamics, the interpretation of the increase of surface area as the increase of entropy should not lead to relate the surface gravity $\kappa$ to temperature. $\kappa$ behaves as a term that represents the attractive force due to gravity. The Hawking radiation may still occur, but the black hole does not get warmer as it evaporates. The material evaporation gets faster as the radius decreases due to the curvature effect similar to the Gibbs-Thomson equation in classical thermodynamics, and the black hole does not warm up as it evaporates.

\section{Funding}

There is no funding support.

\section{Data Access Statement}

There is no data to access.

\section{Ethics Statement}

There is no ethical issue.

\section{Conflicts of Interest}

I declare I have no conflict of interest.

\section{References}

[1] Weinhold, F. (1976) Thermodynamics and Geometry. Physics Today, 29, 23-30 https://physicstoday.scitation.org/doi/10.1063/1.3023366 
https://doi.org/10.1063/1.3023366

[2] Ruppeiner, G. (1979) Thermodynamics: A Riemannian Geometric Model. Physical Review A, 20, 1608-1613. https://doi.org/10.1103/PhysRevA.20.1608

https://journals.aps.org/pra/abstract/10.1103/PhysRevA.20.1608

[3] Gilmore, R. (1984) Length and Curvature in the Geometry of Thermodynamics. Physical Review A, 30, 1994-1997. https://doi.org/10.1103/PhysRevA.30.1994 https://journals.aps.org/pra/abstract/10.1103/PhysRevA.30.1994

[4] Ruppeiner, G. (2013) Unitary Thermodynamics from Thermodynamic Geometry. https://arxiv.org/pdf/1310.2566.pdf

[5] Andresen, B. (2015) Metrics and Energy Landscapes in Irreversible Thermodynamics. Entropy, 17, 6304-6317. https://doi.org/10.3390/e17096304 https://www.mdpi.com/1099-4300/17/9/6304

[6] Lee, J., McGough, L. and Safdi, B.R. (2014) Rényi Entropy and Geometry. https://arxiv.org/pdf/1407.8171.pdf

[7] Mandelbrot, B. (1982) The Fractal Geometry of Nature. WH Freeman \& Co, San Francisco. https://onlinelibrary.wiley.com/doi/abs/10.1002/esp.3290080415

[8] Anders, G., Klotsa, D., Ahmed, N.K., Engel, M. and Glotzer, S. (2014) Understanding Shape Entropy through Local Dense Packing. Proceedings of the National Academy of Sciences of the United States of America, 111, E4812-E4821.

https://doi.org/10.1073/pnas.1418159111

https://www.pnas.org/content/111/45/E4812

[9] Schmitz, G.J. (2018) Entropy and Geometric Objects. Entropy, 20, Article No. 453 https://doi.org/10.3390/e20060453 https://www.mdpi.com/1099-4300/20/6/453

[10] Parker, M.C. and Jeynes, C. (2020) Fullerene Stability by Geometrical Thermodynamics. ChemistrySelect, 5, 5-14. https://doi.org/10.1002/slct.201903633 https://chemistry-europe.onlinelibrary.wiley.com/doi/10.1002/slct.201903633

[11] Oddo, L.A. (1992) Global Shape Entropy: A Mathematically Tractable Approach to Building Extraction in Aerial Imagery. The 20 th AIPR Workshop: Computer Vision Applications: Meeting the Challenges, McLean, 17-18 October 1991, 91-101. https://spie.org/Publications/Proceedings/Paper/10.1117/12.58059?SSO=1

[12] Narayanan, K.R. and Srinivasa, A.R. (2012) Shannon-Entropy-Based Nonequilibrium "Entropic" Temperature of a General Distribution. Physical Review E, 85, Article ID: 031151. https://doi.org/10.1103/PhysRevE.85.031151 https://journals.aps.org/pre/abstract/10.1103/PhysRevE.85.031151

[13] Sahyun, M.R.V. (2018) Aesthetics and Entropy III. Aesthetic Measures. Preprints, Article ID: 2018010098. https://www.preprints.org/manuscript/201801.0098/v1

[14] Arnheim, R. (1971) Entropy and Art. University of California Press, Berkeley. https://www.aakkozzll.com/pdf/arnheim.pdf

[15] Casti, J. and Karlqvist, A. (2003) Art and Complexity. JAI Press, Greenwich. https://www.elsevier.com/books/art-and-complexity/casti/978-0-444-50944-4

[16] Burns, K. (2015) Entropy and Optimality in Abstract Art: An Empirical Test of Visual Aesthetics. Journal of Mathematics and the Arts, 9, 77-90.

https://www.tandfonline.com/doi/full/10.1080/17513472.2015.1096738 https://doi.org/10.1080/17513472.2015.1096738

[17] Birkhoff, G.D. (1933) Aesthetic Measure. Harvard University Press, Cambridge.

[18] Kotecky, R., Shlosman, S. and Dobrushin, R. (1992) Wulff Construction: A Global Shape from Local Interaction. Vol. 104, American Mathematical Society, Provi- 
dence. https://bookstore.ams.org/mmono-104

[19] Wouts, M. (2009) Surface Tension in the Dilute Ising Model: The Wulff Construction. Communications in Mathematical Physics, 289, 157-204.

https://link.springer.com/article/10.1007/s00220-009-0782-8

https://doi.org/10.1007/s00220-009-0782-8

[20] Li, H., Zhao, M. and Jiang, Q. (2009) Cohesive Energy of Clusters Referenced by Wulff Construction. The Journal of Physical Chemistry C, 113, 7594-7597

https://pubs.acs.org/doi/abs/10.1021/jp902319z https://doi.org/10.1021/jp902319z

[21] Mitra, M.K., Menon, G.I. and Rajesh, R.J. (2008) Asymptotic Behavior of Inflated Lattice Polygons. Journal of Statistical Physics, 131, 393-404.

https://link.springer.com/article/10.1007/s10955-008-9512-4 https://doi.org/10.1007/s10955-008-9512-4

[22] Winklmann, S. (2006) A Note on the Stability of the Wulff Shape. Archiv der Mathematik, 87, 272-279. https://link.springer.com/article/10.1007/s00013-006-1685-y https://doi.org/10.1007/s00013-006-1685-y

[23] Sekerka, R.F. (2005) Analytical Criteria for Missing Orientations on Three-Dimensional Equilibrium Shapes. Crystal Research and Technology, 40, 291-306.

https://onlinelibrary.wiley.com/doi/abs/10.1002/crat.200410342 https://doi.org/10.1002/crat.200410342

[24] Miracle-Sole, S. (1995) Surface Tension, Step Free Energy, and Facets in the Equilibrium Crystal. Journal of Statistical Physics, 79, 183-214.

https://link.springer.com/article/10.1007/BF02179386

https://doi.org/10.1007/BF02179386

[25] Leibler, S., Singh, R.R.P. and Fisher, M.E. (1987) Thermodynamic Behavior of Two-Dimensional Vesicles. Physical Review Letters, 59, 1989-1992. https://pubmed.ncbi.nlm.nih.gov/10035389/ https://doi.org/10.1103/physrevlett.59.1989

[26] Rajesh, R. and Dhar, D. (2005) Convex Lattice Polygons of Fixed Area with Perimeter-Dependent Weights. Physical Review E, 71, Article ID: 016130.

https://journals.aps.org/pre/abstract/10.1103/PhysRevE.71.016130 https://doi.org/10.1103/PhysRevE.71.016130

[27] Porter, D.A. and Easterling, K.E. (1992) Phase Transformations in Metals and Alloys. Chapman \& Hall, London; New York.

https://www.worldcat.org/title/phase-transformations-in-metals-and-alloys/oclc/62 $\underline{3259187}$

[28] Van der Eeerden, J.P. (1989) Morphology and Surface Structure of Simple Crystals. In: Sunagawa, I., Ed., Morphology and Growth Unit of Crystals, Terra Scientific Publishing Co., Tokyo, 37-47.

https://www.terrapub.co.jp/e-library/mguc/index.html

[29] Sunagawa, I. (1999) Growth and Morphology of Crystals. Forma, 14, 147-166. https://www.academia.edu/6291100/Growth and Morphology of Crystals

[30] Nehrke, G., Reichart, G.J., Van Cappellen, P., Meile, C. and Bijma, J. (2007) Dependence of Calcite Growth Rate and Sr Partitioning on Solution Stoichiometry: Non-Kossel Crystal Growth. Geochimica et Cosmochimica Acta, 71, 2240-2249. https://core.ac.uk/download/pdf/11759888.pdf https://doi.org/10.1016/j.gca.2007.02.002

[31] Gündüz, G. and Gündüz, U. (2005) The Mathematical Analysis of the Structure of Some Songs. Physica A, 357, 565-592. https://doi.org/10.1016/j.physa.2005.03.042 
https://www.sciencedirect.com/science/article/abs/pii/S0378437105003547

[32] Arnheim, R. (1971) Entropy and Art, an Essay on Disorder and Order. University of California Press, Berkeley. https://www.aakkozzll.com/pdf/arnheim.pdf

[33] Sigaki, H.Y.D., Perc, M. and Ribeiro, H.V. (2018) History of Art Paintings through the Lens of Entropy and Complexity. Proceedings of the National Academy of Sciences of the United States of America, 115, E8585-E8594. https://www.pnas.org/content/115/37/E8585/tab-article-info https://doi.org/10.1073/pnas.1800083115

[34] Hyde, S., Andersson, S., Larsson, K., Blum, Z., Landh, T., Lidin, S. and Ninham, B.W. (1997) The Language of Shape. Elsevier Science, Amsterdam. https://www.elsevier.com/books/the-language-of-shape/hyde/978-0-444-81538-5

[35] Gündüz, G. and Gündüz, Y. (2016) A Thermodynamical View on Asset Pricing. International Review of Financial Analysis, 47, 310-327.

https://www.sciencedirect.com/science/article/abs/pii/S1057521916000144 https://doi.org/10.1016/j.irfa.2016.01.013

[36] Gündüz, G. and Gündüz, A. (2017) Viscoelasticity and Pattern Formations in Stock Market Indices. The European Physical Journal B, 90, Article No. 104

https://link.springer.com/article/10.1140/epjb/e2017-70711-x https://doi.org/10.1140/epjb/e2017-70711-x

[37] Gündüz, G. (2018) Pattern Formation in Time Series Systems Due to Viscoelastic Behavior: Case Studies in Uniform Distribution, Normal Distribution, Stock Market Index, and Music. International Journal of Modern Physics C, 29, Article ID: 1850085. https://doi.org/10.1142/S0129183118500857 https://www.worldscientific.com/doi/10.1142/S0129183118500857

[38] Gündüz, G. and Gündüz, Y. (2010) Viscoelastic Behavior of Stock Indices. Physica $A$, 389, 5776-5784. https://doi.org/10.1016/j.physa.2010.09.010 https://www.sciencedirect.com/science/article/abs/pii/S0378437110007909

[39] Christodoulou, D. (1971) Reversible and Irreversible Transformations in Black-Hole Physics. Physical Review Letters, 25, 1596-1597.

https://journals.aps.org/prl/abstract/10.1103/PhysRevLett.25.1596

https://doi.org/10.1103/PhysRevLett.25.1596

[40] Bekenstein, J.D. (1973) Black Holes and Entropy. Physical Review D, 7, 2333-2346 https://journals.aps.org/prd/abstract/10.1103/PhysRevD.7.2333 https://doi.org/10.1103/PhysRevD.7.2333

[41] Bardeen, J.M., Carter, B. and Hawking, S. (1973) The Four Laws of Black Hole Mechanics. Communications in Mathematical Physics, 31, 161-170.

[42] Hawking, S.W. (1975) Particle Creation by Black Boles. Communications in Mathematical Physics, 43, 199-220. https://doi.org/10.1007/BF02345020

https://link.springer.com/article/10.1007/BF02345020

[43] Hawking, S.W. (1974) Black Hole Explosions? Nature, 248, 30-31 https://www.nature.com/articles/248030a0 https://doi.org/10.1038/248030a0

[44] Smarr, L. (1973) Mass Formula for Kerr Black Holes. Physical Review Letters, 30, 71-73. https://journals.aps.org/prl/abstract/10.1103/PhysRevLett.30.71 https://doi.org/10.1103/PhysRevLett.30.71

[45] Davies, P.C.W. (1978) Thermodynamics of Black Holes. Reports on Progress in Physics, 41, 1313-1355. https://doi.org/10.1088/0034-4885/41/8/004 https://iopscience.iop.org/article/10.1088/0034-4885/41/8/004/pdf 
[46] Bekenstein, J.D. (2001) The Limits of Information. Studies in History and Philosophy of Science Part B, 32, 511-524. https://doi.org/10.1016/S1355-2198(01)00020-X

[47] Fulling, S.A. (1973) Nonuniqueness of Canonical Field Quantization in Riemannian Space-Time. Physical Review D, 7, 2850-2862.

https://journals.aps.org/prd/abstract/10.1103/PhysRevD.7.2850

https://doi.org/10.1103/PhysRevD.7.2850

[48] Davies, P.C.W. (1975) Scalar Production in Schwarzschild and Rindler Metrics. Journal of Physics A: Mathematical and General, 8, 609-616. https://iopscience.iop.org/article/10.1088/0305-4470/8/4/022/pdf https://doi.org/10.1088/0305-4470/8/4/022

[49] Gibbons, G.W. and Hawking, S.W. (1977) Cosmological Event Horizons, Thermodynamics, and Particle Creation. Physical Review D, 15, 2738-2751 https://journals.aps.org/prd/abstract/10.1103/PhysRevD.15.2738 https://doi.org/10.1103/PhysRevD.15.2738

[50] Padmanabhan, T. (2010) Thermodynamical Aspects of Gravity: New Insights. Reports on Progress in Physics, 73, Article ID: 046901 https://iopscience.iop.org/article/10.1088/0034-4885/73/4/046901/pdf https://doi.org/10.1088/0034-4885/73/4/046901

[51] Jacobson, T. and Parentani, R. (2003) Horizon Entropy. Foundations of Physics, 33, 323-348. https://link.springer.com/article/10.1023/A:1023785123428 https://doi.org/10.1023/A:1023785123428

[52] Davies, P.C.W. and Taylor, J.G. (1974) Do Black Holes Really Explode? Nature, 250, 37-38. https://www.nature.com/articles/250037a0 https://doi.org/10.1038/250037a0

[53] Helfer, A.D. (2003) Do Black Holes Radiate? Reports on Progress in Physics, 66, 943-1008. https://iopscience.iop.org/article/10.1088/0034-4885/66/6/202/pdf https://doi.org/10.1088/0034-4885/66/6/202

[54] Padmanabhan, T. (2004) Entropy of Static Spacetimes and Microscopic Density of States. Classical and Quantum Gravity, 21, 4485-4494

https://iopscience.iop.org/article/10.1088/0264-9381/21/18/013/pdf https://doi.org/10.1088/0264-9381/21/18/013

[55] 't Hooft, G. (2009) Dimensional Reduction in Quantum Gravity. arXiv:gr-qc/9310026. https://arxiv.org/abs/gr-qc/9310026

[56] Susskind, L. (1994) The World as a Hologram. Journal of Mathematical Physics, 36, 6377-6396. https://arxiv.org/abs/hep-th/9409089 https://doi.org/10.1063/1.531249

[57] Chen, H.Z., Fisher, Z., Hernandez, J., Myers, R.C. and Ruan, S.M. (2020) Information Flow in Black Hole Evaporation. Journal of High Energy Physics, 2020, Article No. 152. https://link.springer.com/content/pdf/10.1007/JHEP03(2020)152.pdf https://doi.org/10.1007/JHEP03(2020)152

[58] Verlinde, E. (2011) On the Origin of Gravity and the Laws of Newton. Journal of High Energy Physics, 2011, Article No. 29. https://link.springer.com/content/pdf/10.1007/JHEP04(2011)029.pdf https://doi.org/10.1007/JHEP04(2011)029

[59] Smolin, L. (2010) Newtonian Gravity in Loop Quantum Gravity. https://arxiv.org/pdf/1001.3668.pdf 


\section{Appendix A}
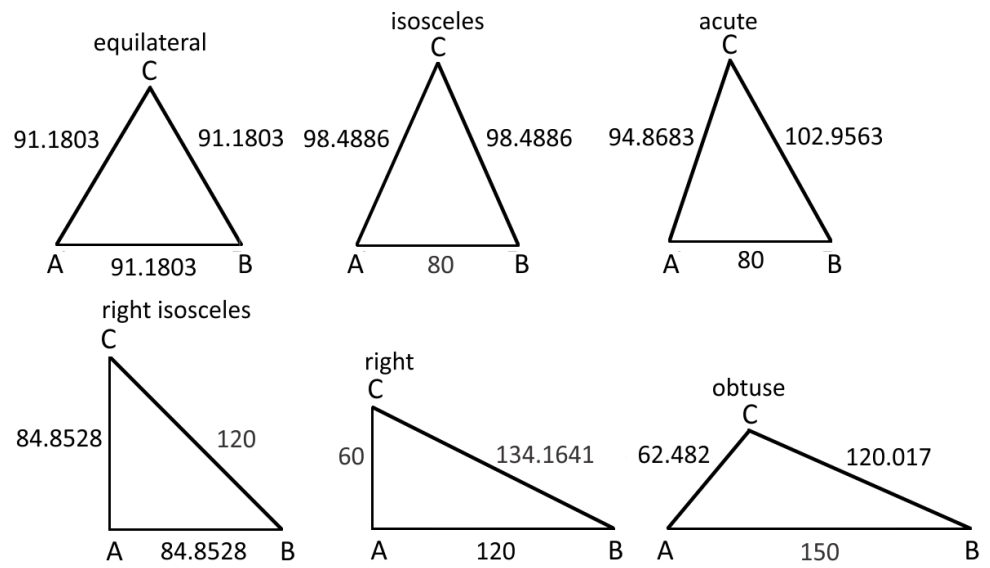

Figure A1. Triangles.

\section{Appendix B}
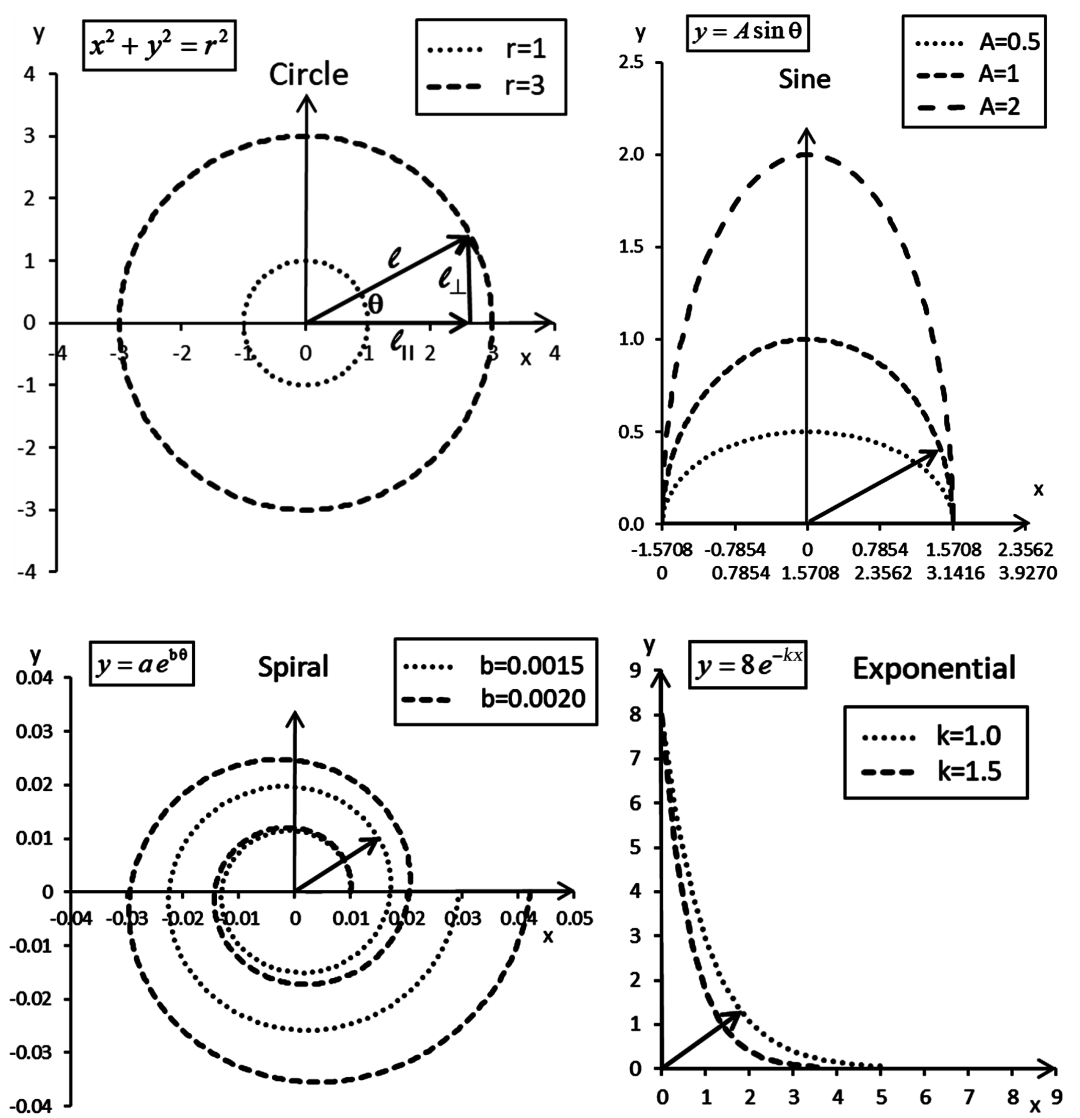

Figure B1. The formulas, pictures and parameters of the curves studied. 